Citation: Özdemir Y. \& Nalbant K. G. (2018), A Real Personnel Selection Problem Using The Generalized Choquet Integral Methodology, BMIJ, (2018), 6(2): 694-716 doi: http://dx.doi.org/10.15295/bmij.v6i2.270

\title{
A REAL PERSONNEL SELECTION PROBLEM USING THE GENERALIZED CHOQUET INTEGRAL METHODOLOGY
}

Yavuz ÖZDEMIR ${ }^{1}$

Kemal Gökhan NALBANT ${ }^{2}$
Received Date (Başvuru Tarihi): 26/07/2018

Accepted Date (Kabul Tarihi): 30/08/2018

Published Date (Yayın Tarihi): 02/09/2018

\begin{abstract}
The main objective in the selection of personnel is to select the most appropriate candidate for a job. Personnel selection for human resources management is a very important issue. The aim of this paper is to determine the best-performing personnel for promotion using an application of a Multi Criteria Decision Making (MCDM) method, generalized Choquet integral, to a real personnel selection problem of a case study in Turkey and 17 alternatives are ranked according to personnel selection criteria (22 subcriteria are classified under 5 main criteria). The main contribution of this paper is to determine the interdependency among main criteria and subcriteria, the nonlinear relationship among them and the environmental uncertainties while selecting personnel alternatives using the generalized Choquet integral method with the experts' view. To the authors' knowledge, this will be the first study which uses the generalized Choquet Integral methodology for human resources.
\end{abstract}

Keywords: Personnel Selection, Human Resources, Multi Criteria Decision Making (MCDM), Generalized Choquet Integral.

JEL Codes: M51, D81, C020

\section{GENELLEŞTIRILMIŞ CHOQUET INTEGRAL METODOLOJISİ KULLANILARAK GERÇEK BİR PERSONEL SEÇIM PROBLEMI}

ÖZ

Personel seçiminde temel amaç, bir işe en uygun olan adayı seçmektir. Insan kaynakları yönetimi için personel seçimi çok önemli bir konudur. Bu çalışmanın amacı, Türkiye'de gerçek bir personel seçim problemi örnek olay çalışmasına Çok Kriterli Karar Verme (ÇKKV) yöntemi olan genelleştirilmiş Choquet integrali uygulamasinu kullanarak terfi için en iyi performansa sahip personeli belirlemek ve bu 17 alternatifi personel seçim kriterlerine göre sıralamaktır (22 alt kriter 5 ana kriter altında sinıflandırllmıştır). Bu makalenin ana katklsı, uzmanların görüşleriyle genelleştirilmiş Choquet integral yöntemini kullanarak personel alternatiflerini seçerken, ana kriterler ve alt kriterler arasındaki bağımlılığın, aralarındaki doğrusal olmayan ilişkinin ve çevresel belirsizliklerin belirlenmesidir. Yazarların bilgisine göre bu çalışma, insan kaynakları için genelleştirilmiş Choquet integral metodolojisini kullanan ilk çalışma olacakttr.

Anahtar Kelimeler: Personel Seçimi, İnsan Kaynakları, Çok Kriterli Karar Verme (ÇKKV), Genelleştirilmiş Choquet Integral.

JEL Kodlart: M51, D81, C020

\footnotetext{
${ }^{1}$ Dr., İstanbul Sabahattin Zaim University, yavuzytu @ gmail.com

${ }^{2}$ Dr., Yildiz Technical University, kgokhannalbant@gmail.com
} 


\section{INTRODUCTION}

Recruitment in companies is a business process. Training, experience and personal characteristics are important qualities for personnel to be recruited. The personal characteristics of the personnel to be recruited determine the attitude to his work and his compatibility with colleagues. If personal characteristics of the candidate to be recruited match the duties and responsibilities requied for the job, the requirements of the job are fulfilled at that level (Nalbant, 2017).

The most important task of the Human Resources Department is to support management by using human resources effectively. It is the responsibility of this department to plan all operations related to personnel. This department is also responsible for the workplace to adapt the new methods to the operation, which will increase productivity. In addition, for this department, it is a task to provide consulting and internal communication to increase employee effectiveness (Nalbant, 2017).

Personnel selection is the most important factor in human resources management. Personnel Selection arises from the needs of the company's employees and aims to select the most suitable person for an open position. In order for the selection process to begin, the number of candidates must be greater than the numbers of employees required. At the end of the process, the most appropriate person to meet the criteria is selected among the applicants (Nalbant, 2017).

The rest of the paper is organized as follows; in the Section 2, the literature review is given. Choquet Integral methodology is explained in Section 3, and in Section 4, the problem definition about personnel selection problem is mentioned. In Section 5, Choquet Integral methodology is applied for this problem. Moreover, the results are given in Section 6. Finally, the results are evaluated in Section 7.

\section{LITERATURE REVIEW}

Selection or prioritization of the best alternative from a range of available alternatives based on multiple criteria is usually called as multi-criteria decision-making (MCDM) (Ozdemir and Basligil, 2016). MCDM methods can be used to solve personnel selection problem. There have been many studies on the selection of personnel using MCDM methods in the literature (Chen, 2000; Afshari et al., 2010; Kelemenis and Askounis, 2010; Boran et al., 2011; Rashidi et al., 2011; Baležentis et al., 2012; Kabak et. al., 2012; Roy and Misra, 2012; Yu et al., 2013; Violeta and Turskis, 2014; Karabasevic, 2015). 
In the literature, Choquet Integral has been studied extensively. Grabisch and Roubens (2000) gave an application of the Choquet Integral in MCDM. Their explanation avoided the listing of all properties of the Choquet integral and its relation with ordinary aggregation operations. Moreover, they investigated the connection with game theory and how to identify in an experimental problem the fuzzy measure modeling the decision maker's behaviour in detail. But their work didn't aim to cover all the range of a MCDM problem, only to address the aggregation step. Karsak (2005) applied Choquet Integral for robot selection problem. Because Choquet Integral can take into account interaction among robot attributes. This method was used to determine the best robot. Mazaud et al. (2007) proposed a feature selection method by using Choquet Integral. He aimed to enhance model interpretability by selecting best important features among a list extracted from images and to replace expert selection by automatically selecting a suitable set of features. Tseng et al. (2009) proposed an evaluation framework using Analytic Network Process and Choquet Integral for optimal supplier selection. They found that their evaluation framework was simple and reasonable to identify the primary criteria influencing the supply chain management strategy (SCMS).

Tan and Chen (2010) proposed an intuitionistic fuzzy Choquet Integral for multi-criteria decision making and gave an example to evaluate the results. They applied fuzzy measures because it is not suitable to aggregate the criteria by traditional aggregation operators. Demirel et al. (2010) used Choquet Integral for a warehouse location selection problem because conventional approaches to warehouse location selection problem tend to be less effective and included some subcriteria because of the hierarchical structure of the problem. Bebčáková et al. (2011) proposed the Generalized Partial Goals Method (GPGM) by integrating the Choquet Integral. In their method, the aggregation with weighted average was replaced by aggregation with the Choquet Integral. Also, they discussed the application of fuzzified Choquet Integral to multiple criteria evaluation and proposed a new method for fuzzy measure construction. Wu et al. (2013) proposed intuitionistic fuzzy aggregation functions by integrating IFS Theory with the Choquet Integral and gave their aggregation properties in fuzzy MCDM. He aimed to show the integration properties of intuitionistic fuzzy-valued Choquet integrals and to avoid improper applications of this type of Choquet Integral in MCDM. Li et al. (2013) introduced a new method using the Choquet Integral for hotel selection to benefit tourism managers. With the help of this method, managers can allocate their limited resources to improve the aspects of their hotels and they can have more confidence in their decision making while reducing the investment risk. 
Gomesa et al. (2015) proposed a methodology using the Choquet Integral in order to improve systems usability. So, the results pointed out which are the most impacting metrics for the university's intranet system and leaded to most relevant constructs that minimize the costs to improve the usability of the system. Nia et al. (2016) proposed a methodology by fuzzy Choquet Integral for a supplier selection problem. Their method was applied to a manufacturing company to assess the applicability of the method. Besides, their method can be used for real world problems that contain fuzziness or interacting decision criteria. Demirel et al. (2017) proposed an approach by using Choquet Integral for underground natural gas storage location selection. Because, large scale storage of natural gas is very significant and they found out their method is useful and practical for the location selection. Büyüközkan et al. (2018) proposed an integrated intuitionistic fuzzy Choquet Integral approach on public bus technologies selection. They found that dependencies among decision criteria affect the selection process of the most sustainable urban transportation system.

The aim of this study is to select the best personnel to be promoted in a company according to the prioritized personnel selection criteria which were defined in the recent research (Ozdemir et al., 2017). In the recent research, personnel selection criteria were determined and prioritized by using Consistent Fuzzy Preference Relations (CFPR), which is one of MCDM methods (Ozdemir et al., 2017). However, CFPR methodology can only prioritize personnel selection criteria and it cannot select the best alternative. Additionally, no study has been carried out so far, using Choquet Integral for personnel selection in the literature. This is the first study that uses this method in personnel selection area.

\section{CHOQUET INTEGRAL}

Choquet integral is a sort of general averaging operator that can represent the notions of importance of a criteria and interactions among criteria. A set of values of importance is composed of the values of a fuzzy measure. The success of a Choquet integral depends on an appropriate representation of fuzzy measures, which captures the importance of individual criterion or their combination (Demirel et al., 2010).

Relationship between trapezoidal fuzzy numbers and degrees of linguistic importance on a nine-linguistic-term scale can be seen from Table 1 . 
Table 1: Relationship between Trapezoidal Fuzzy Numbers and Degrees of Linguistic Importance on a Nine-Linguistic-Term Scale

\begin{tabular}{|c|c|c|c|c|}
\hline \multicolumn{2}{|c|}{ Low/high Levels } & \multicolumn{2}{|c|}{ Degrees of Importance } & \multirow{2}{*}{$\begin{array}{l}\text { Trapezoidal fuzzy } \\
\text { numbers }\end{array}$} \\
\hline Label & Linguistic Terms & Label & Linguistic Terms & \\
\hline EL & Extra low & EU & Extra unimportant & $(0,0,0,0)$ \\
\hline $\mathrm{VL}$ & Very low & VU & Very unimportant & $(0.00,0.01,0.02,0.07)$ \\
\hline $\mathrm{L}$ & Low & $\mathrm{U}$ & Unimportant & $(0.04,0.10,0.18,0.23)$ \\
\hline SL & Slightly low & SU & Slightly unimportant & $(0.17,0.22,0.36,0.42)$ \\
\hline $\mathrm{M}$ & Middle & $\mathrm{M}$ & Middle & $(0.32,0.41,0.58,0.65)$ \\
\hline $\mathrm{SH}$ & Slightly high & SI & Slightly important & $(0.58,0.63,0.80,0.86)$ \\
\hline $\mathrm{H}$ & High & HI & High important & $(0.72,0.78,0.92,0.97)$ \\
\hline $\mathrm{VH}$ & Very high & VI & Very important & $(0.93,0.98,0.98,1.00)$ \\
\hline $\mathrm{EH}$ & Extra high & EI & Extra important & $(1,1,1,1)$ \\
\hline
\end{tabular}

Source: Delgado, M., Herrera, F., Herrera-Viedma, E. \& Martnez, L. (1998). Combining numerical and linguistic information in group decision making. Information Sciences, 107, 177-194.

The methodology consists of eight steps (Chen and Tzeng, 2001; Chiou et al., 2005; Demirel et al., 2010; Meyer and Roubens, 2006; Tsai and Lu, 2006):

Step-1: Given criteria $i$, respondents' linguistic preferences for the degree of importance, perceived performance levels of alternative personnel, and tolerance zone are surveyed.

Step-2: In view of the compatibility between perceived performance levels and the tolerance zone, trapezoidal fuzzy numbers are used to quantify all linguistic terms. Given respondent $t$ and criteria $i$, linguistic terms for the degree of importance is parameterized by $\tilde{A}_{i}^{t}=\left(a_{i 1}^{t}, a_{i 2}^{t}, a_{i 3}^{t}, a_{i 4}^{t}\right)$, perceived performance levels by $\tilde{p}_{i}^{t}=\left(p_{i 1}^{t}, p_{i 2}^{t}, p_{i 3}^{t}, p_{i 4}^{t}\right)$, and the tolerance zone by $\tilde{e}_{i}^{t}=\left(e_{i 1 L}^{t}, e_{i 2 L}^{t}, e_{i 3 U}^{t}, e_{i 4 U}^{t}\right)$.

Step-3: $\tilde{A}_{i}^{t}, \tilde{p}_{i}^{t}$ and $\tilde{e}_{i}^{t}$ into $\tilde{A}_{i}$ and $\tilde{e}_{i}$ are averaged respectively using (1).

$$
\widetilde{A}_{i}=\frac{\sum_{t=1}^{k} \tilde{A}_{i}^{t}}{k}=\left(\frac{\sum_{t=1}^{k} a_{i 1}^{t}}{k}, \frac{\sum_{t=1}^{k} a_{i 2}^{t}}{k}, \frac{\sum_{t=1}^{k} a_{i 3}^{t}}{k}, \frac{\sum_{t=1}^{k} a_{i 4}^{t}}{k}\right)
$$

Step-4: The value of each criteria are normalized by using (2).

$$
\tilde{f}_{i}=\underset{\alpha \in[0,1]}{\| \bar{f}_{i}^{\alpha}}=\underset{\substack{\alpha \in[0,1] \\ \alpha}}{-}\left[f_{i \alpha}^{+}\right]
$$

where $f_{i} \in F(S)$ is a fuzzy-valued function. $\tilde{F}(S)$ is the set of all fuzzy-valued functions $f, f_{i}^{\alpha}=\left[f_{i \alpha}^{-}, f_{i \alpha}^{+}\right]=\frac{\bar{p}_{i}^{\alpha}-\bar{e}_{i}^{\alpha}+[1,1]}{2}, \bar{p}_{i}^{\alpha}$ and $\bar{e}_{i}^{\alpha}$ are $\alpha$-level cuts of $\tilde{p}_{i}$ and $\tilde{e}_{i}$ for all $\alpha=[0,1]$.

Step-5: The value of dimension $j$ is found by using (3). 
$\left.(C) \int \tilde{f} d \tilde{g}=\|(C) \int f_{\alpha}^{-} d g_{\alpha=[0,1]}^{-},(C) \int f_{\alpha}^{+} d g_{\alpha}^{+}\right\rfloor$

where $\quad \bar{g}_{i}: P(S) \rightarrow I\left(R^{+}\right), \quad \bar{g}_{i}=\left[g_{i}^{-}, g_{i}^{+}\right], \quad \bar{g}_{i}^{\alpha}=\left[g_{i, \alpha}^{-}, g_{i, \alpha}^{+}\right], \quad \bar{f}_{i}: S \rightarrow I\left(R^{+}\right), \quad$ and $f_{i}=\left[f_{i}^{-}, f_{i}^{+}\right]$for $\mathrm{i}=1,2,3, \ldots, \mathrm{nj}$.

To be able to calculate this value, a $\lambda$ value and the fuzzy measures $g(A(i)), i=1,2,3, \ldots, n$ are needed. These are obtained from the following (4-6).

$$
\begin{aligned}
& \mathrm{g}(\mathrm{A}(\mathrm{n}))=\mathrm{g}(\{\mathrm{s}(\mathrm{n})\})=\mathrm{gn}, \\
& \mathrm{g}(\mathrm{A}(\mathrm{i}))=\mathrm{gi}+\mathrm{g}(\mathrm{A}(\mathrm{i}+1))+\lambda \operatorname{gig}(\mathrm{A}(\mathrm{i}+1)), \text { where } 1 \leq \mathrm{i}<\mathrm{n} \\
& 1=g(S)=\left\{\begin{array}{lr}
1 / \lambda\left\{\prod_{i=1}^{n}\left[1+\lambda g\left(A_{i}\right)\right]-1\right\} & \text { if } \lambda \neq 0 \\
\sum_{i=1}^{n} g\left(A_{i}\right) & \text { if } \lambda=0,
\end{array}\right.
\end{aligned}
$$

where, $A i \cap A j=\varnothing$ for all $i, j=1,2,3, \ldots, n$ and $i \neq j$, and $\lambda \in(-1, \infty]$.

Let $\mu$ be a fuzzy measure on (I,P(I)) and an application $f: I \rightarrow \mathfrak{R}^{+}$. The Choquet integral of $\mathrm{f}$ with respect to $\mu$ is defined by:

$$
(C) \int_{I} f d \mu=\sum_{i=1}^{n}(f(\sigma(i))-f(\sigma(i-1))) \mu\left(A_{(i)}\right)
$$

where $\sigma$ is a permutation of the indices in order to have $f(\sigma(1)) \leq \ldots \leq f(\sigma(n)), A_{(i)}=\{\sigma(i), \ldots, \sigma(n)\}$ and $x f(\sigma(0))=0$, by convention.

Under rather general assumptions over the set of alternatives $\mathrm{X}$, and over the weak orders $\succeq_{i}$, there exists a unique fuzzy measure $\mu$ over I such that (Demirel et al., 2010):

$$
\forall x, y \in X, x \succeq y \Leftrightarrow u(x) \geq u(y)
$$

where

$$
u(x)=\sum_{i=1}^{n}\left[u_{(i)}\left(x_{(i)}\right)-u_{(i-1)}\left(x_{(i-1)}\right)\right] \mu\left(A_{(i)}\right),
$$

which is simply the aggregation of the monodimensional utility functions using the Choquet integral with respect to $\mu$. 
Step-6: All dimensional performance levels of the personnel alternatives into overall performance levels are aggregated, using a hierarchical process applying the two-stage aggregation process of the generalized Choquet integral (10). The overall performance levels yield a fuzzy number, $\tilde{V}$.

$$
\begin{array}{cc}
\text { main }_{\text {critera }}^{(1)}=(C) \int f d g \\
\text { main }_{\text {criteria }}(m) \\
=(C) \int f d g
\end{array}
$$

Step-7: It is assumed that the membership of $\tilde{V}$ is $\mu \mathrm{v}(\mathrm{x})$; defuzzy the fuzzy number $\tilde{V}$ into a crisp value $\mathrm{v}$ using (11) and a comparison of the overall performance levels of alternative personnel is made.

$$
F(\tilde{A})=\frac{v_{1}+v_{2}+v_{3}+v_{4}}{4}
$$

Step-8: Weak and advantageous criteria among the personnel alternatives are compared by using (1) (Demirel et al., 2010).

\section{PROBLEM DEFINITION}

In this section, personnel selection problem is studied and the personnel are prioritized using Choquet Integral methodology according to personnel selection criteria (Table 2). In this study, a company is chosen for the personnel selection problem which is located in Istanbul, Turkey. The company intends to promote one of its engineers to a chief-engineer position. 5 main criteria, 22 subcriteria and 17 alternatives are determined by three evaluators of the academicians and the managers of the company (Ozdemir et al., 2017). For this personnel selection problem, decision criteria (main and subcriteria) can be seen in Table 2. 
Table 2: Personnel Selection Criteria

\begin{tabular}{|c|c|c|c|}
\hline \multicolumn{2}{|r|}{ Main Criteria } & \multicolumn{2}{|r|}{ Subcriteria } \\
\hline \multirow{3}{*}{ M1 } & \multirow{3}{*}{ ACTIVITY } & S11 & Productive Activity \\
\hline & & S12 & Auxiliary Activity \\
\hline & & S13 & Inefficient Activity \\
\hline \multirow{3}{*}{ M2 } & \multirow{3}{*}{ FEE } & S21 & Fee Paid \\
\hline & & S22 & Payable Fee \\
\hline & & S23 & Requested Fee \\
\hline \multirow{6}{*}{ M3 } & \multirow{6}{*}{ EDUCATION } & S31 & Education Status \\
\hline & & S32 & Foreign Languages \\
\hline & & S33 & Certificates \\
\hline & & S34 & Job Experience \\
\hline & & S35 & Technology Usage \\
\hline & & S36 & Lifelong Learning \\
\hline \multirow{6}{*}{ M4 } & \multirow{6}{*}{ INTERNAL FACTORS } & S41 & Self-Confidence \\
\hline & & S42 & Take Initiative \\
\hline & & S43 & Analytic Thinking \\
\hline & & S44 & Leadership \\
\hline & & S45 & Productivity \\
\hline & & S46 & Decision Making / Problem Solving \\
\hline \multirow{4}{*}{ M5 } & \multirow{4}{*}{ BUSINESS FACTORS } & S51 & Compatible with the Team / Communication \\
\hline & & S52 & Teamwork Skills \\
\hline & & S53 & Finishing Work on Time \\
\hline & & S54 & Business Discipline \\
\hline
\end{tabular}

Source: Ozdemir, Y., Nalbant, K. G. \& Basligil, H. (2017). Evaluation of personnel selection criteria using Consistent Fuzzy Preference Relations. Operations Research and Information Engineering, 2, 1-6.

Among the criteria, "Activity" contains productive, auxiliary and inefficient activities of personnel. "Productive Activity" subcriteria means activities which provide a large amount of good for the firm. "Auxiliary Activity" subcriteria means activities which provide a small amount of good for the firm. "Inefficient Activity" subcriteria means activities which provide a tiny amount of good for the firm.

"Fee" criteria means the fee charged by the employee during his / her working period. "Fee Paid" subcriteria means price that directly paid. "Payable Fee" subcriteria means price that should be paid for personnel. "Requested Fee" subcriteria means price that personnel requested.

"Education" criteria means education level of the personnel. "Education Status" subcriteria means level of education of personnel. "Foreign Languages" subcriteria means any language other than that spoken by the personnel. "Certificates" subcriteria means official documents taken by the personnel. "Job Experince" subcriteria means short-term or long-term experiences of personnel. "Technology Usage" subcriteria means technology knowledge of 
personnel to solve the problems. "Lifelong Learning" subcriteria means continuously development and improvement of the knowledge and skills needed for the work.

"Internal Factors" criteria means inner features that a personnel to be recruited has. "Self-Confidence" subcriteria means feeling of trust in one's abilities and qualities. "Take Initiative" subcriteria means the ability to assess and initiate things independently. "Analytic Thinking" subcriteria means the ability to solve problems quickly and effectively. "Leadership" subcriteria means the action of leading a group of people or an organization. "Productivity" subcriteria means a measure of the efficiency of a personnel. "Decision Making / Problem Solving" subcriteria means the action of making important decision and finding solutions for difficult issues.

"Business Factors" criteria means outer features that a personnel to be recruited has. "Compatible with the Team / Communication" subcriteria means the imparting of information by speaking or writing. "Teamwork Skills" subcriteria means the willingness of a group of people to work together to achieve a common aim. "Finishing Work on Time" subcriteria means to be punctual. "Business Discipline" subcriteria means the practices that help a business grow.

\section{APPLICATION: A REAL CASE STUDY}

All experts are asked to determine using the evaluation scale in Table 1. The importance of main and subcriteria, their tolerance zones are generated and each alternative's linguistic evaluation are given in Table 3 by evaluators. To measure the linguistic terms in Table 3, trapezoidal numbers are used. The combined tolerance zones are determined by the following way; the first two values of the lower linguistic value of a tolerance zone in Table 3 are combined with the last two values of the upper linguistic value of a tolerance zone. Consider the tolerance zone $[\mathrm{M}, \mathrm{H}]$. The corresponding numerical values of $\mathrm{M}$ and $\mathrm{H}$ are $(0.32,0.41$, $0.58,0.65)$ and $(0.72,0.78,0.92,0.97)$, respectively. Then the combined tolerance zone is $(0.32$, $0.41,0.92,0.97)$. Table 4 shows the compromised evaluations of the three evaluators.

The evaluation results are obtained by the generalized Choquet Integral for $\alpha=0$ and for $\alpha=1$ in Table 5 and Table 7, respectively. "Importance" column are the lowest and the highest value of the "Importance". For example, the trapezoidal fuzzy numbers for "High" is $(0.72,0.78,0.92,0.97)$, and the "Importance" for that criteria is obtained as $(0.72,0.97)$. For the subcriteria, (2) is used while (3) is for the main criteria. For example, the value [0.375, 0.825] of "A1 and subcriteria S21" is obtained in the following way: 


$$
f, f_{i}^{\alpha}=\left[f_{i \alpha}^{-}, f_{i \alpha}^{+}\right]=\frac{[0.72,0.07]-[0,97,0.32]+[1,1]}{2}=[0.375,0.825]
$$

By solving the following equation for $\lambda$ (Table 6 , Table 8 ), the fuzzy weightsg $\left(\mathrm{A}_{(\mathrm{i})}\right)$, $\mathrm{i}=1,2, . ., \mathrm{n}$ are obtained using (4-6) as follows:

$$
1=g(S)=\frac{1}{\lambda}\{[(1+0.72 \lambda)(1+0.72 \lambda)(1+0.93 \lambda)]-1\}
$$

That is,

$\lambda=-0.9939$.

$\mathrm{g}\left(\mathrm{A}_{(1)}\right)=\mathrm{g}_{1}=0.72$,

$\mathrm{g}\left(\mathrm{A}_{(2)}\right)=\mathrm{g}_{2}+\mathrm{g}\left(\mathrm{A}_{(1)}\right)+\lambda \mathrm{g}_{2} \mathrm{~g}\left(\mathrm{~A}_{(1)}\right)=0.92$,

$\mathrm{g}\left(\mathrm{A}_{(3)}\right)=\mathrm{g}_{3}+\mathrm{g}\left(\mathrm{A}_{(2)}\right)+\lambda \mathrm{g}_{3} \mathrm{~g}\left(\mathrm{~A}_{(2)}\right)=1.00$,

Fuzzy weights and $\lambda$ values are calculated by the generalized Choquet Integral in Table 6 and Table 8.

The aggregated Choquet integral values for the main criteria "M2" are calculated as in the following (7-10): In Table 5 and 7, the fuzzy measures for "A1" according to the main criteria are $(0.439,0.483,0.755,0.825)$. The first fuzzy measure 0.439 is calculated using the Table 5 and 6 as:

$$
\{[1 \times 0.36]+[0.92 x(0.36-0.375)]+[0.72 x(0.375-0.465)]\}=0.439
$$

The first overall alternative value for "A1" $(0.464)$ in Table 9 is obtained in that way:

$$
\begin{gathered}
\{[1 \times 0.343]+[1 \times(0.357-0.343)]+[1 \times(0.439-0.357)]+[0.98 \times(0.462-0.439)] \\
+[0.93 \times(0.464-0.462)]\}=0.464
\end{gathered}
$$


Table 3: Importance of Criteria, The Tolerance Zones and Each Personnel's Linguistic Evaluation

\begin{tabular}{|c|c|c|c|c|c|c|c|c|c|c|c|c|c|c|c|c|c|c|c|}
\hline \multirow{2}{*}{ Criteria } & \multirow{2}{*}{$\begin{array}{l}\text { Importance } \\
\text { of criteria }\end{array}$} & \multirow{2}{*}{$\begin{array}{c}\text { Tolerance } \\
\text { Zone }\end{array}$} & \multicolumn{17}{|c|}{ Linguistic evaluation } \\
\hline & & & A1 & A2 & A3 & A4 & A5 & A6 & A7 & A8 & A9 & A10 & A11 & A12 & A13 & A14 & A15 & A16 & A17 \\
\hline M1 & VI & & & & & & & & & & & & & & & & & & \\
\hline S11 & VI & {$[\mathrm{H}, \mathrm{EH}]$} & $\mathrm{VH}$ & EH & VH & VH & VH & VH & VH & VH & EH & VH & VH & VH & $\mathrm{H}$ & $\mathrm{H}$ & $\mathrm{H}$ & $\mathrm{H}$ & $\mathrm{H}$ \\
\hline S12 & VI & {$[\mathrm{SH}, \mathrm{VH}]$} & $\mathrm{H}$ & $\mathrm{VH}$ & $\mathrm{H}$ & VH & $\mathrm{H}$ & $\mathrm{H}$ & $\mathrm{H}$ & $\mathrm{H}$ & $\mathrm{VH}$ & $\mathrm{H}$ & $\mathrm{H}$ & $\mathrm{H}$ & $\mathrm{H}$ & $\mathrm{H}$ & SH & $\mathrm{SH}$ & $\mathrm{SH}$ \\
\hline S13 & VI & {$[\mathrm{M}, \mathrm{VH}]$} & VH & $\mathrm{H}$ & $\mathrm{VH}$ & $\mathrm{H}$ & VH & VH & $\mathrm{H}$ & SH & VH & $\mathrm{H}$ & $\mathrm{H}$ & SH & M & M & M & $\mathrm{M}$ & $\mathrm{M}$ \\
\hline M2 & $\mathrm{HI}$ & & & & & & & & & & & & & & & & & & \\
\hline S21 & $\mathrm{HI}$ & {$[\mathrm{M}, \mathrm{H}]$} & $\mathrm{H}$ & $\mathrm{H}$ & $\mathrm{H}$ & $\mathrm{H}$ & $\mathrm{H}$ & $\mathrm{H}$ & SH & SH & $\mathrm{H}$ & $\mathrm{H}$ & $\mathrm{SH}$ & $\mathrm{SH}$ & $\mathrm{M}$ & $\mathrm{M}$ & $\mathrm{M}$ & $\mathrm{M}$ & $\mathrm{M}$ \\
\hline S22 & $\mathrm{HI}$ & {$[\mathrm{SH}, \mathrm{VH}]$} & $\mathrm{VH}$ & VH & $\mathrm{VH}$ & VH & $\mathrm{H}$ & $\mathrm{H}$ & $\mathrm{H}$ & $\mathrm{H}$ & $\mathrm{VH}$ & $\mathrm{H}$ & $\mathrm{H}$ & $\mathrm{H}$ & $\mathrm{H}$ & SH & SH & $\mathrm{SH}$ & $\mathrm{SH}$ \\
\hline S23 & VI & {$[\mathrm{M}, \mathrm{VH}]$} & $\mathrm{H}$ & $\mathrm{VH}$ & $\mathrm{H}$ & $\mathrm{H}$ & $\mathrm{VH}$ & $\mathrm{H}$ & SH & SH & $\mathrm{H}$ & SH & SH & SH & SH & SH & $\mathrm{M}$ & $\mathrm{SH}$ & $\mathrm{M}$ \\
\hline M3 & SI & & & & & & & & & & & & & & & & & & \\
\hline S31 & SI & {$[\mathrm{SL}, \mathrm{VH}]$} & $\mathrm{H}$ & $\mathrm{H}$ & $\mathrm{VH}$ & $\mathrm{H}$ & $\mathrm{H}$ & $\mathrm{SH}$ & $\mathrm{SH}$ & $\mathrm{M}$ & $\mathrm{H}$ & $\mathrm{H}$ & $\mathrm{SH}$ & $\mathrm{M}$ & $\mathrm{M}$ & $\mathrm{M}$ & $\mathrm{SH}$ & $\mathrm{M}$ & SL \\
\hline S32 & SI & {$[\mathrm{SL}, \mathrm{VH}]$} & SH & $\mathrm{H}$ & $\mathrm{H}$ & SH & SH & SH & SH & $\mathrm{M}$ & $\mathrm{VH}$ & SH & SH & $\mathrm{M}$ & SL & SL & SL & SL & SL \\
\hline S33 & SU & {$[\mathrm{SL}, \mathrm{SH}]$} & $\mathrm{M}$ & $\mathrm{SH}$ & $\mathrm{SH}$ & $\mathrm{SH}$ & $\mathrm{M}$ & $\mathrm{M}$ & $\mathrm{M}$ & $\mathrm{SH}$ & $\mathrm{SH}$ & $\mathrm{M}$ & $\mathrm{M}$ & $\mathrm{SH}$ & $\mathrm{SH}$ & $\mathrm{M}$ & SL & SL & SL \\
\hline S34 & $\mathrm{M}$ & {$[\mathrm{M}, \mathrm{H}]$} & $\mathrm{H}$ & $\mathrm{H}$ & $\mathrm{H}$ & $\mathrm{H}$ & $\mathrm{H}$ & $\mathrm{SH}$ & SH & $\mathrm{SH}$ & $\mathrm{H}$ & SH & SH & SH & SH & SH & $\mathrm{M}$ & $\mathrm{SH}$ & $\mathrm{M}$ \\
\hline S35 & $\mathrm{M}$ & {$[\mathrm{L}, \mathrm{M}]$} & SL & $\mathrm{M}$ & SL & SL & SL & $\mathrm{M}$ & SL & SL & $\mathrm{M}$ & SL & SL & SL & $\mathrm{L}$ & $\mathrm{L}$ & SL & $\mathrm{L}$ & $\mathrm{L}$ \\
\hline S36 & $\mathrm{M}$ & {$[\mathrm{L}, \mathrm{M}]$} & SL & $\mathrm{M}$ & $M$ & $\mathrm{M}$ & $M$ & $\mathrm{M}$ & $\mathrm{M}$ & SL & SL & $M$ & $\mathrm{M}$ & SL & SL & $\mathrm{L}$ & $\mathrm{L}$ & $\mathrm{L}$ & $\mathrm{L}$ \\
\hline M4 & $\mathrm{HI}$ & & & & & & & & & & & & & & & & & & \\
\hline S41 & SU & {$[\mathrm{SL}, \mathrm{SH}]$} & $\mathrm{M}$ & $\mathrm{SH}$ & SH & $\mathrm{SH}$ & SH & $\mathrm{M}$ & $\mathrm{M}$ & $\mathrm{M}$ & SH & SH & $\mathrm{M}$ & M & $\mathrm{M}$ & $\mathrm{M}$ & SL & SL & SL \\
\hline $\mathrm{S} 42$ & SI & {$[\mathrm{M}, \mathrm{VH}]$} & $\mathrm{VH}$ & $\mathrm{H}$ & $\mathrm{H}$ & $\mathrm{H}$ & $\mathrm{H}$ & $\mathrm{VH}$ & $\mathrm{H}$ & $\mathrm{H}$ & $\mathrm{H}$ & $\mathrm{H}$ & $\mathrm{H}$ & $\mathrm{H}$ & $\mathrm{H}$ & $\mathrm{H}$ & $\mathrm{M}$ & $\mathrm{SH}$ & $\mathrm{M}$ \\
\hline S43 & VI & {$[\mathrm{SH}, \mathrm{VH}]$} & $\mathrm{VH}$ & VH & $\mathrm{H}$ & $\mathrm{H}$ & VH & $\mathrm{VH}$ & $\mathrm{H}$ & $\mathrm{H}$ & $\mathrm{H}$ & $\mathrm{H}$ & $\mathrm{H}$ & $\mathrm{H}$ & SH & SH & SH & SH & SH \\
\hline S44 & SI & {$[\mathrm{M}, \mathrm{VH}]$} & $\mathrm{SH}$ & $\mathrm{H}$ & $\mathrm{VH}$ & $\mathrm{VH}$ & $\mathrm{H}$ & SH & SH & $\mathrm{SH}$ & $\mathrm{VH}$ & $\mathrm{SH}$ & SH & SH & $\mathrm{SH}$ & $\mathrm{M}$ & $\mathrm{M}$ & $\mathrm{M}$ & $\mathrm{M}$ \\
\hline S45 & $\mathrm{HI}$ & {$[\mathrm{M}, \mathrm{H}]$} & $\mathrm{H}$ & $\mathrm{SH}$ & $\mathrm{SH}$ & $\mathrm{SH}$ & $\mathrm{SH}$ & $\mathrm{H}$ & $\mathrm{SH}$ & $\mathrm{SH}$ & $\mathrm{H}$ & $\mathrm{H}$ & $\mathrm{SH}$ & $\mathrm{SH}$ & $\mathrm{M}$ & $\mathrm{M}$ & $\mathrm{SH}$ & $\mathrm{SH}$ & $\mathrm{M}$ \\
\hline S46 & $\mathrm{HI}$ & {$[\mathrm{SH}, \mathrm{VH}]$} & $\mathrm{H}$ & $\mathrm{SH}$ & $\mathrm{H}$ & $\mathrm{H}$ & $\mathrm{H}$ & $\mathrm{H}$ & $\mathrm{H}$ & $\mathrm{H}$ & SH & $\mathrm{H}$ & $\mathrm{H}$ & $\mathrm{H}$ & $\mathrm{H}$ & SH & $\mathrm{SH}$ & $\mathrm{SH}$ & $\mathrm{SH}$ \\
\hline M5 & $M$ & & & & & & & & & & & & & & & & & & \\
\hline S51 & $\mathrm{M}$ & {$[\mathrm{M}, \mathrm{H}]$} & $\mathrm{SH}$ & $\mathrm{H}$ & $\mathrm{H}$ & $\mathrm{H}$ & $\mathrm{H}$ & $\mathrm{SH}$ & $\mathrm{SH}$ & $\mathrm{SH}$ & $\mathrm{H}$ & $\mathrm{SH}$ & $\mathrm{SH}$ & $\mathrm{SH}$ & $\mathrm{SH}$ & $\mathrm{SH}$ & $\mathrm{M}$ & $\mathrm{M}$ & $\mathrm{M}$ \\
\hline S52 & SI & {$[\mathrm{SL}, \mathrm{VH}]$} & $\mathrm{H}$ & $\mathrm{H}$ & $\mathrm{H}$ & $\mathrm{VH}$ & $\mathrm{H}$ & $\mathrm{H}$ & $\mathrm{H}$ & $\mathrm{H}$ & $\mathrm{SH}$ & $\mathrm{H}$ & $\mathrm{H}$ & $\mathrm{H}$ & $\mathrm{H}$ & SH & $M$ & SH & SL \\
\hline S53 & SU & {$[\mathrm{L}, \mathrm{SH}]$} & $\mathrm{SH}$ & $\mathrm{SH}$ & $\mathrm{M}$ & $\mathrm{M}$ & $\mathrm{SH}$ & $\mathrm{M}$ & $\mathrm{M}$ & SL & $\mathrm{M}$ & $\mathrm{M}$ & $\mathrm{M}$ & SL & $\mathrm{L}$ & $\mathrm{L}$ & $\mathrm{L}$ & $\mathrm{L}$ & SL \\
\hline S54 & $\mathrm{M}$ & {$[\mathrm{M}, \mathrm{H}]$} & $\mathrm{H}$ & $\mathrm{H}$ & $\mathrm{H}$ & SH & SH & $\mathrm{H}$ & $\mathrm{SH}$ & SH & $\mathrm{H}$ & $\mathrm{H}$ & $\mathrm{SH}$ & SH & SH & SH & $M$ & $M$ & $\mathrm{M}$ \\
\hline
\end{tabular}


Table 4: Compromised Evaluations of Three Evaluators

\begin{tabular}{|c|c|c|c|c|c|c|c|c|c|c|c|c|c|c|c|c|c|c|c|c|c|c|c|c|c|c|c|c|c|c|c|c|}
\hline \multirow{3}{*}{$\begin{array}{c}\text { Criteria } \\
\text { M1 } \\
\text { S11 } \\
\end{array}$} & \multicolumn{4}{|c|}{ Importance } & \multicolumn{4}{|c|}{ Combined T. Zone } & \multicolumn{4}{|c|}{ A1 } & \multicolumn{4}{|c|}{ A2 } & \multicolumn{4}{|c|}{ A 3} & \multicolumn{4}{|c|}{ A4 } & \multirow{2}{*}{\multicolumn{4}{|c|}{ A5 }} & \multicolumn{4}{|c|}{ A6 } \\
\hline & 0.93 & 0.98 & 0.98 & 1 & & & & & & & & & \multirow[b]{2}{*}{1} & \multirow[b]{2}{*}{1} & \multirow[b]{2}{*}{1} & & \multirow[b]{2}{*}{0.93} & \multirow[b]{2}{*}{0.98} & \multirow[b]{2}{*}{0.98} & & \multirow[b]{2}{*}{0.93} & \multirow[b]{2}{*}{0.98} & & & & & & & & & & \\
\hline & 0.93 & 0.98 & 0.98 & 1 & 0.72 & 0.78 & 1 & 1 & 0.93 & 0.98 & 0.98 & 1 & & & & & & & & & & & 0.98 & 1 & 0.93 & 0.98 & 0.98 & 1 & 0.93 & 0.98 & 0.98 & 1 \\
\hline S12 & 0.93 & 0.98 & 0.98 & 1 & 0.58 & 0.63 & 0.98 & 1 & 0.72 & 0.78 & 0.92 & 0.97 & 0.93 & 0.98 & 0.98 & 1 & 0.72 & 0.78 & 0.92 & 0.97 & 0.93 & 0.98 & 0.98 & 1 & 0.72 & 0.78 & 0.92 & 0.97 & 0.72 & 0.78 & 0.92 & 0.97 \\
\hline $\mathrm{S} 13$ & 0.93 & 0.98 & 0.98 & 1 & 0.32 & 0.41 & 0.98 & 1 & 0.93 & 0.98 & 0.98 & 1 & 0.72 & 0.78 & 0.92 & 0.97 & 0.93 & 0.98 & 0.98 & 1 & 0.72 & 0.78 & 0.92 & 0.97 & 0.93 & 0.98 & 0.98 & 1 & 0.93 & 0.98 & 0.98 & 1 \\
\hline M2 & 0.72 & 0.78 & 0.92 & 0.97 & & & & & & & & & & & & & & & & & & & & & & & & & & & & \\
\hline $\mathrm{S} 21$ & 0.72 & 0.78 & 0.92 & 0.97 & 0.32 & 0.41 & 0.92 & 0.97 & 0.72 & 0.78 & 0.92 & 0.97 & 0.72 & 0.78 & 0.92 & 0.97 & 0.72 & 0.78 & 0.92 & 0.97 & 0.72 & 0.78 & 0.92 & 0.97 & 0.72 & 0.78 & 0.92 & 0.97 & 0.72 & 0.78 & 0.92 & 0.97 \\
\hline $\mathrm{S} 22$ & 0.72 & 0.78 & 0.92 & 0.97 & 0.58 & 0.63 & 0.98 & 1 & 0.93 & 0.98 & 0.98 & 1 & 0.93 & 0.98 & 0.98 & 1 & 0.93 & 0.98 & 0.98 & 1 & 0.93 & 0.98 & 0.98 & 1 & 0.72 & 0.78 & 0.92 & 0.97 & 0.72 & 0.78 & 0.92 & 0.97 \\
\hline $\mathrm{S} 23$ & 0.93 & 0.98 & 0.98 & 1 & 0.32 & 0.41 & 0.98 & 1 & 0.72 & 0.78 & 0.92 & 0.97 & 0.93 & 0.98 & 0.98 & 1 & 0.72 & 0.78 & 0.92 & 0.97 & 0.72 & 0.78 & 0.92 & 0.97 & 0.93 & 0.98 & 0.98 & 1 & 0.72 & 0.78 & 0.92 & 0.97 \\
\hline M3 & 0.58 & 0.63 & 0.8 & 0.86 & & & & & & & & & & & & & & & & & & & & & & & & & & & & \\
\hline S31 & 0.58 & 0.63 & 0.8 & 0.86 & 0.17 & 0.22 & 0.98 & 1 & 0.72 & 0.78 & 0.92 & 0.97 & 0.72 & 0.78 & 0.92 & 0.97 & 0.93 & 0.98 & 0.98 & 1 & 0.72 & 0.78 & 0.92 & 0.97 & 0.72 & 0.78 & 0.92 & 0.97 & 0.58 & 0.63 & 0.8 & 0.86 \\
\hline $\mathrm{S} 32$ & 0.58 & 0.63 & 0.8 & 0.86 & 0.17 & 0.22 & 0.98 & 1 & 0.58 & 0.63 & 0.8 & 0.86 & 0.72 & 0.78 & 0.92 & 0.97 & 0.72 & 0.78 & 0.92 & 0.97 & 0.58 & 0.63 & 0.8 & 0.86 & 0.58 & 0.63 & 0.8 & 0.86 & 0.58 & 0.63 & 0.8 & 0.86 \\
\hline S33 & 0.17 & 0.22 & 0.36 & 0.42 & 0.17 & 0.22 & 0.8 & 0.86 & 0.32 & 0.41 & 0.58 & 0.65 & 0.58 & 0.63 & 0.8 & 0.86 & 0.58 & 0.63 & 0.8 & 0.86 & 0.58 & 0.63 & 0.8 & 0.86 & 0.32 & 0.41 & 0.58 & 0.65 & 0.32 & 0.41 & 0.58 & 0.65 \\
\hline S34 & 0.32 & 0.41 & 0.58 & 0.65 & 0.32 & 0.41 & 0.92 & 0.97 & 0.72 & 0.78 & 0.92 & 0.97 & 0.72 & 0.78 & 0.92 & 0.97 & 0.72 & 0.78 & 0.92 & 0.97 & 0.72 & 0.78 & 0.92 & 0.97 & 0.72 & 0.78 & 0.92 & 0.97 & 0.58 & 0.63 & 0.8 & 0.86 \\
\hline S35 & 0.32 & 0.41 & 0.58 & 0.65 & 0.04 & 0.1 & 0.58 & 0.65 & 0.17 & 0.22 & 0.36 & 0.42 & 0.32 & 0.41 & 0.58 & 0.65 & 0.17 & 0.22 & 0.36 & 0.42 & 0.17 & 0.22 & 0.36 & 0.42 & 0.17 & 0.22 & 0.36 & 0.42 & 0.32 & 0.41 & 0.58 & 0.65 \\
\hline S36 & 0.32 & 0.41 & 0.58 & 0.65 & 0.04 & 0.1 & 0.58 & 0.65 & 0.17 & 0.22 & 0.36 & 0.42 & 0.32 & 0.41 & 0.58 & 0.65 & 0.32 & 0.41 & 0.58 & 0.65 & 0.32 & 0.41 & 0.58 & 0.65 & 0.32 & 0.41 & 0.58 & 0.65 & 0.32 & 0.41 & 0.58 & 0.65 \\
\hline M4 & 0.72 & 0.78 & 0.92 & 0.97 & & & & & & & & & & & & & & & & & & & & & & & & & & & & \\
\hline S41 & 0.17 & 0.22 & 0.36 & 0.42 & 0.17 & 0.22 & 0.8 & 0.86 & 0.32 & 0.41 & 0.58 & 0.65 & 0.58 & 0.63 & 0.8 & 0.86 & 0.58 & 0.63 & 0.8 & 0.86 & 0.58 & 0.63 & 0.8 & 0.86 & 0.58 & 0.63 & 0.8 & 0.86 & 0.32 & 0.41 & 0.58 & 0.65 \\
\hline $\mathrm{S} 42$ & 0.58 & 0.63 & 0.8 & 0.86 & 0.32 & 0.41 & 0.98 & 1 & 0.93 & 0.98 & 0.98 & 1 & 0.72 & 0.78 & 0.92 & 0.97 & 0.72 & 0.78 & 0.92 & 0.97 & 0.72 & 0.78 & 0.92 & 0.97 & 0.72 & 0.78 & 0.92 & 0.97 & 0.93 & 0.98 & 0.98 & 1 \\
\hline S43 & 0.93 & 0.98 & 0.98 & 1 & 0.58 & 0.63 & 0.98 & 1 & 0.93 & 0.98 & 0.98 & 1 & 0.93 & 0.98 & 0.98 & 1 & 0.72 & 0.78 & 0.92 & 0.97 & 0.72 & \begin{tabular}{|l|}
0.78 \\
\end{tabular} & 0.92 & 0.97 & 0.93 & 0.98 & 0.98 & 1 & 0.93 & 0.98 & 0.98 & 1 \\
\hline S44 & 0.58 & 0.63 & 0.8 & 0.86 & 0.32 & 0.41 & 0.98 & 1 & 0.58 & 0.63 & 0.8 & 0.86 & 0.72 & 0.78 & 0.92 & 0.97 & 0.93 & 0.98 & 0.98 & 1 & 0.93 & 0.98 & 0.98 & 1 & 0.72 & 0.78 & 0.92 & 0.97 & 0.58 & 0.63 & 0.8 & 0.86 \\
\hline S45 & 0.72 & 0.78 & 0.92 & 0.97 & 0.32 & 0.41 & 0.92 & 0.97 & 0.72 & 0.78 & 0.92 & 0.97 & 0.58 & 0.63 & 0.8 & 0.86 & 0.58 & 0.63 & 0.8 & 0.86 & 0.58 & 0.63 & 0.8 & 0.86 & 0.58 & 0.63 & 0.8 & 0.86 & 0.72 & 0.78 & 0.92 & 0.97 \\
\hline S46 & 0.72 & 0.78 & 0.92 & 0.97 & 0.58 & 0.63 & 0.98 & 1 & 0.72 & 0.78 & 0.92 & 0.97 & 0.58 & 0.63 & 0.8 & 0.86 & 0.72 & 0.78 & 0.92 & 0.97 & 0.72 & 0.78 & 0.92 & 0.97 & 0.72 & 0.78 & 0.92 & 0.97 & 0.72 & 0.78 & 0.92 & 0.97 \\
\hline M5 & 0.32 & 0.41 & 0.58 & 0.65 & & & & & & & & & & & & & & & & & & & & & & & & & & & & \\
\hline S51 & 0.32 & 0.41 & 0.58 & 0.65 & 0.32 & 0.41 & 0.92 & 0.97 & 0.58 & 0.63 & 0.8 & 0.86 & 0.72 & 0.78 & 0.92 & 0.97 & 0.72 & 0.78 & 0.92 & 0.97 & 0.72 & 0.78 & 0.92 & 0.97 & 0.72 & 0.78 & 0.92 & 0.97 & 0.58 & 0.63 & 0.8 & 0.86 \\
\hline S52 & 0.58 & 0.63 & 0.8 & 0.86 & 0.17 & 0.22 & 0.98 & 1 & 0.72 & 0.78 & 0.92 & 0.97 & 0.72 & 0.78 & 0.92 & 0.97 & 0.72 & 0.78 & 0.92 & 0.97 & 0.93 & 0.98 & 0.98 & 1 & 0.72 & 0.78 & 0.92 & 0.97 & 0.72 & 0.78 & 0.92 & 0.97 \\
\hline S53 & 0.17 & 0.22 & 0.36 & 0.42 & 0.04 & 0.1 & 0.8 & 0.86 & 0.58 & 0.63 & 0.8 & 0.86 & 0.58 & 0.63 & 0.8 & 0.86 & 0.32 & 0.41 & 0.58 & 0.65 & 0.32 & 0.41 & 0.58 & 0.65 & 0.58 & 0.63 & 0.8 & 0.86 & 0.32 & 0.41 & 0.58 & 0.65 \\
\hline S54 & 0.32 & 0.41 & 0.58 & 0.65 & 0.32 & 0.41 & 0.92 & 0.97 & 0.72 & 0.78 & 0.92 & 0.97 & 0.72 & 0.78 & 0.92 & 0.97 & 0.72 & 0.78 & 0.92 & 0.97 & 0.58 & 0.63 & 0.8 & 0.86 & 0.58 & 0.63 & 0.8 & 0.86 & 0.72 & 0.78 & 0.92 & 0.97 \\
\hline
\end{tabular}


Table 5: Evaluation Using The Generalized Choquet Integral for $\alpha=0$

\begin{tabular}{|c|c|c|c|c|c|c|c|c|c|c|c|c|c|c|c|c|c|c|c|c|}
\hline \multirow{3}{*}{$\begin{array}{c}\text { Criteria } \\
\text { M1 }\end{array}$} & \multicolumn{2}{|c|}{ Importance of criteria } & \multicolumn{18}{|c|}{$\left[\mathrm{fi}^{-}, \mathrm{fi}^{+}\right]$} \\
\hline & & & \multicolumn{2}{|c|}{ A1 } & \multicolumn{2}{|c|}{ A2 } & \multicolumn{2}{|c|}{ A3 } & \multicolumn{2}{|c|}{ A4 } & \multicolumn{2}{|c|}{ A5 } & \multicolumn{2}{|c|}{ A6 } & \multicolumn{2}{|c|}{ A7 } & \multicolumn{2}{|c|}{ A8 } & \multicolumn{2}{|c|}{ A9 } \\
\hline & & & 0.464 & 0.840 & 0.497 & 0.825 & 0.464 & 0.840 & 0.464 & 0.825 & 0.464 & 0.840 & 0.464 & 0.840 & 0.458 & 0.825 & 0.457 & 0.770 & 0.498 & 0.840 \\
\hline S11 & 0.93 & 1 & 0.465 & 0.64 & 0.5 & 0.64 & 0.465 & 0.64 & 0.465 & 0.64 & 0.465 & 0.64 & 0.465 & 0.64 & 0.465 & 0.64 & 0.465 & 0.64 & 0.5 & 0.64 \\
\hline S12 & 0.93 & 1 & 0.36 & 0.695 & 0.465 & 0.71 & 0.36 & 0.695 & 0.465 & 0.71 & 0.36 & 0.695 & 0.36 & 0.695 & 0.36 & 0.695 & 0.36 & 0.695 & 0.465 & 0.71 \\
\hline S13 & 0.93 & 1 & 0.465 & 0.84 & 0.36 & 0.825 & 0.465 & 0.84 & 0.36 & 0.825 & 0.465 & 0.84 & 0.465 & 0.84 & 0.36 & 0.825 & 0.29 & 0.77 & 0.465 & 0.84 \\
\hline M2 & & & 0.439 & 0.825 & 0.464 & 0.840 & 0.439 & 0.825 & 0.439 & 0.825 & 0.458 & 0.840 & 0.371 & 0.825 & 0.343 & 0.770 & 0.343 & 0.770 & 0.439 & 0.825 \\
\hline S21 & 0.72 & 0.97 & 0.375 & 0.825 & 0.375 & 0.825 & 0.375 & 0.825 & 0.375 & 0.825 & 0.375 & 0.825 & 0.375 & 0.825 & 0.305 & 0.77 & 0.305 & 0.77 & 0.375 & 0.825 \\
\hline S22 & 0.72 & 0.97 & 0.465 & 0.71 & 0.465 & 0.71 & 0.465 & 0.71 & 0.465 & 0.71 & 0.36 & 0.695 & 0.36 & 0.695 & 0.36 & 0.695 & 0.36 & 0.695 & 0.465 & 0.71 \\
\hline $\mathrm{S} 23$ & 0.93 & 1 & 0.36 & 0.825 & 0.465 & 0.84 & 0.36 & 0.825 & 0.36 & 0.825 & 0.465 & 0.84 & 0.36 & 0.825 & 0.29 & 0.77 & 0.29 & 0.77 & 0.36 & 0.825 \\
\hline M3 & & & 0.343 & 0.891 & 0.363 & 0.899 & 0.419 & 0.911 & 0.352 & 0.892 & 0.348 & 0.892 & 0.316 & 0.844 & 0.306 & 0.844 & 0.265 & 0.795 & 0.419 & 0.911 \\
\hline S31 & 0.58 & 0.86 & 0.36 & 0.9 & 0.36 & 0.9 & 0.465 & 0.915 & 0.36 & 0.9 & 0.36 & 0.9 & 0.29 & 0.845 & 0.29 & 0.845 & 0.16 & 0.74 & 0.36 & 0.9 \\
\hline S32 & 0.58 & 0.86 & 0.29 & 0.845 & 0.36 & 0.9 & 0.36 & 0.9 & 0.29 & 0.845 & 0.29 & 0.845 & 0.29 & 0.845 & 0.29 & 0.845 & 0.16 & 0.74 & 0.465 & 0.915 \\
\hline S33 & 0.17 & 0.42 & 0.23 & 0.74 & 0.36 & 0.845 & 0.36 & 0.845 & 0.36 & 0.845 & 0.23 & 0.74 & 0.23 & 0.74 & 0.23 & 0.74 & 0.36 & 0.845 & 0.36 & 0.845 \\
\hline S34 & 0.32 & 0.65 & 0.375 & 0.825 & 0.375 & 0.825 & 0.375 & 0.825 & 0.375 & 0.825 & 0.375 & 0.825 & 0.305 & 0.77 & 0.305 & 0.77 & 0.305 & 0.77 & 0.375 & 0.825 \\
\hline S35 & 0.32 & 0.65 & 0.26 & 0.69 & 0.335 & 0.805 & 0.26 & 0.69 & 0.26 & 0.69 & 0.26 & 0.69 & 0.335 & 0.805 & 0.26 & 0.69 & 0.26 & 0.69 & 0.335 & 0.805 \\
\hline S36 & 0.32 & 0.65 & 0.26 & 0.69 & 0.335 & 0.805 & 0.335 & 0.805 & 0.335 & 0.805 & 0.335 & 0.805 & 0.335 & 0.805 & 0.335 & 0.805 & 0.26 & 0.69 & 0.26 & 0.69 \\
\hline M4 & & & 0.462 & 0.838 & 0.457 & 0.833 & 0.421 & 0.840 & 0.421 & 0.840 & 0.458 & 0.833 & 0.462 & 0.838 & 0.360 & 0.817 & 0.360 & 0.817 & 0.425 & 0.841 \\
\hline S41 & & 0.42 & & & 0.36 & 0.845 & 0.36 & 0.845 & 0.36 & 0.845 & 0.36 & 0.845 & 0.23 & 0.74 & 0.23 & 0.74 & 0.23 & 0.74 & 0.36 & 0.845 \\
\hline S42 & & 0.86 & 0.465 & 0.84 & 0.36 & 0.825 & 0.36 & 0.825 & 0.36 & 0.825 & 0.36 & 0.825 & 0.465 & 0.84 & 0.36 & 0.825 & 0.36 & 0.825 & 0.36 & 0.825 \\
\hline S43 & & 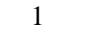 & 0.465 & & 0.465 & 0.71 & 0.36 & 0.695 & 0.36 & 0.695 & 0.465 & 0.71 & 0.465 & 0.71 & 0.36 & 0.695 & 0.36 & 0.695 & 0.36 & 0.695 \\
\hline S44 & & & & & 0.36 & 0.825 & 0.465 & 0.84 & 0.465 & 0.84 & 0.36 & 0.825 & 0.29 & 0.77 & 0.29 & 0.77 & 0.29 & 0.77 & 0.465 & 0.84 \\
\hline S45 & & 0.97 & 0.375 & 0.825 & 0.305 & 0.77 & 0.305 & 0.77 & 0.305 & 0.77 & 0.305 & 0.77 & 0.375 & 0.825 & 0.305 & 0.77 & 0.305 & 0.77 & 0.375 & 0.825 \\
\hline S46 & 0.72 & 0.97 & 0.36 & 0.695 & 0.29 & 0.64 & 0.36 & 0.695 & 0.36 & 0.695 & 0.36 & 0.695 & 0.36 & 0.695 & 0.36 & 0.695 & 0.36 & 0.695 & 0.29 & 0.64 \\
\hline M5 & & & 0.357 & 0.897 & 0.369 & 0.898 & 0.360 & 0.889 & 0.407 & 0.901 & 0.357 & 0.897 & 0.348 & 0.888 & 0.332 & 0.884 & 0.327 & 0.881 & 0.335 & 0.842 \\
\hline S51 & 0.32 & 0.65 & 0.305 & 0.77 & 0.375 & 0.825 & 0.375 & 0.825 & 0.375 & 0.825 & 0.375 & 0.825 & 0.305 & 0.77 & 0.305 & 0.77 & 0.305 & 0.77 & 0.375 & 0.825 \\
\hline S52 & 0.58 & 0.86 & 0.36 & 0.9 & 0.36 & 0.9 & 0.36 & 0.9 & 0.465 & 0.915 & 0.36 & 0.9 & 0.36 & 0.9 & 0.36 & 0.9 & 0.36 & 0.9 & 0.29 & 0.845 \\
\hline S53 & 0.17 & 0.42 & 0.36 & 0.91 & 0.36 & 0.91 & 0.23 & 0.805 & 0.23 & 0.805 & 0.36 & 0.91 & 0.23 & 0.805 & 0.23 & 0.805 & 0.155 & 0.69 & 0.23 & 0.805 \\
\hline S54 & 0.32 & 0.65 & 0.375 & 0.825 & 0.375 & 0.825 & 0.375 & 0.825 & 0.305 & 0.77 & 0.305 & 0.77 & 0.375 & 0.825 & 0.305 & 0.77 & 0.305 & 0.77 & 0.375 & 0.825 \\
\hline
\end{tabular}


Yavuz ÖZDEMIR \& Kemal Gökhan NALBANT

Table 6: Fuzzy Weights $g\left(\mathrm{~A}_{(\mathrm{i})}\right)$ and $\lambda$ Values for $\alpha=0$

\begin{tabular}{|c|c|c|c|c|c|c|c|c|c|c|c|c|c|c|c|c|c|c|}
\hline & \multicolumn{18}{|c|}{$\left[\mathrm{gi}^{-}=\mathrm{g}\left(\mathrm{A}_{(\mathrm{i})}\right), \mathrm{gi}^{+}=\mathrm{g}\left(\mathrm{A}_{(\mathrm{i})}\right)\right]$} \\
\hline & \multicolumn{2}{|c|}{$\mathrm{A} 1$} & \multicolumn{2}{|c|}{ A2 } & \multicolumn{2}{|c|}{ A3 } & \multicolumn{2}{|c|}{ A4 } & \multicolumn{2}{|c|}{ A5 } & \multicolumn{2}{|r|}{ A6 } & \multicolumn{2}{|r|}{ A7 } & \multicolumn{2}{|r|}{ A8 } & \multicolumn{2}{|r|}{ A9 } \\
\hline$\lambda$ & -0.9997 & -1 & -0.9997 & -1 & -0.9997 & -1 & -0.9997 & -1 & -0.9997 & -1 & -0.9997 & -1 & -0.9997 & -1 & -0.9997 & -1 & -0.9997 & -1 \\
\hline & 0.93 & 1.00 & 0.93 & 1.00 & 0.93 & 1.00 & 0.93 & 1.00 & 0.93 & 1.00 & 0.93 & 1.00 & 0.93 & 1.00 & 0.93 & 1.00 & 0.93 & 1.00 \\
\hline & 1.00 & 1.00 & 1.00 & 1.00 & 1.00 & 1.00 & 1.00 & 1.00 & 1.00 & 1.00 & 1.00 & 1.00 & 1.00 & 1.00 & 1.00 & 1.00 & 1.00 & 1.00 \\
\hline & 1.00 & 1.00 & 1.00 & 1.00 & 1.00 & 1.00 & 1.00 & 1.00 & 1.00 & 1.00 & 1.00 & 1.00 & 1.00 & 1.00 & 1.00 & 1.00 & 1.00 & 1.00 \\
\hline \multirow[t]{4}{*}{$\lambda$} & -0.9939 & -1 & -0.9939 & -1 & -0.9939 & -1 & -0.9939 & -1 & -0.9939 & -1 & -0.9939 & -1 & -0.9939 & -1 & -0.9939 & -1 & -0.9939 & -1 \\
\hline & 0.72 & 0.97 & 0.72 & 1.00 & 0.72 & 0.97 & 0.72 & 0.97 & 0.93 & 1.00 & 0.72 & 0.97 & 0.72 & 0.97 & 0.72 & 0.97 & 0.72 & 0.97 \\
\hline & 0.92 & 1.00 & 0.98 & 1.00 & 0.92 & 1.00 & 0.92 & 1.00 & 0.98 & 1.00 & 0.92 & 1.00 & 0.92 & 1.00 & 0.92 & 1.00 & 0.92 & 1.00 \\
\hline & 1.00 & 1.00 & 1.00 & 1.00 & 1.00 & 1.00 & 1.00 & 1.00 & 1.00 & 1.00 & 1.00 & 1.00 & 1.00 & 1.00 & 1.00 & 1.00 & 1.00 & 1.00 \\
\hline \multirow[t]{7}{*}{$\lambda$} & -0.9408 & -0.9995 & -0.9408 & -0.9995 & $\begin{array}{l}-0.9408 \\
\end{array}$ & -0.9995 & \begin{tabular}{|c|}
-0.9408 \\
\end{tabular} & -0.9995 & -0.9408 & \begin{tabular}{|l|}
-0.9995 \\
\end{tabular} & $\begin{array}{l}-0.9408 \\
\end{array}$ & -0.9995 & $\begin{array}{r}-0.9408 \\
\end{array}$ & $\begin{array}{l}-0.9995 \\
\end{array}$ & -0.9408 & -0.9995 & -0.9408 & -0.9995 \\
\hline & 0.32 & 0.86 & 0.32 & 0.86 & 0.58 & 0.86 & 0.32 & 0.86 & 0.32 & 0.86 & 0.32 & 0.86 & 0.32 & 0.86 & 0.17 & 0.42 & 0.58 & 0.86 \\
\hline & 0.73 & 0.98 & 0.44 & 0.98 & 0.73 & 0.98 & 0.44 & 0.98 & 0.73 & 0.98 & 0.54 & 0.98 & 0.54 & 0.98 & 0.44 & 0.80 & 0.73 & 0.98 \\
\hline & 0.91 & 0.99 & 0.78 & 0.99 & 0.78 & 0.99 & 0.78 & 0.99 & 0.83 & 0.99 & 0.70 & 0.99 & 0.83 & 0.99 & 0.63 & 0.97 & 0.78 & 0.99 \\
\hline & 0.96 & 1.00 & 0.93 & 1.00 & 0.93 & 1.00 & 0.86 & 1.00 & 0.96 & 1.00 & 0.90 & 1.00 & 0.96 & 1.00 & 0.76 & 1.00 & 0.93 & 1.00 \\
\hline & 0.99 & 1.00 & 0.97 & 1.00 & 0.97 & 1.00 & 0.97 & 1.00 & 0.99 & 1.00 & 0.99 & 1.00 & 0.99 & 1.00 & 0.92 & 1.00 & 0.97 & 1.00 \\
\hline & 1.00 & 1.00 & 1.00 & 1.00 & 1.00 & 1.00 & 1.00 & 1.00 & 1.00 & 1.00 & 1.00 & 1.00 & 1.00 & 1.00 & 1.00 & 1.00 & 1.00 & 1.00 \\
\hline \multirow[t]{7}{*}{$\lambda$} & -0.9992 & -1 & -0.9992 & -1 & -0.9992 & -1 & -0.9992 & -1 & -0.9992 & -1 & -0.9992 & -1 & -0.9992 & -1 & -0.9992 & -1 & -0.9992 & -1 \\
\hline & 0.58 & 0.86 & 0.93 & 0.42 & 0.58 & 0.42 & 0.58 & 0.42 & 0.93 & 0.42 & 0.58 & 0.86 & 0.58 & 0.86 & 0.58 & 0.86 & 0.58 & 0.42 \\
\hline & 0.97 & 1.00 & 0.94 & 0.92 & 0.65 & 0.92 & 0.65 & 0.92 & 0.94 & 0.92 & 0.97 & 1.00 & 0.88 & 0.98 & 0.88 & 0.98 & 0.88 & 0.92 \\
\hline & 0.99 & 1.00 & 0.98 & 0.99 & 0.85 & 0.99 & 0.85 & 0.99 & 0.98 & 0.99 & 0.99 & 1.00 & 0.99 & 1.00 & 0.99 & 1.00 & 0.90 & 0.99 \\
\hline & 1.00 & 1.00 & 0.99 & 1.00 & 0.96 & 1.00 & 0.96 & 1.00 & 0.99 & 1.00 & 1.00 & 1.00 & 1.00 & 1.00 & 1.00 & 1.00 & 0.96 & 1.00 \\
\hline & 1.00 & 1.00 & 1.00 & 1.00 & 1.00 & 1.00 & 1.00 & 1.00 & 1.00 & 1.00 & 1.00 & 1.00 & 1.00 & 1.00 & 1.00 & 1.00 & 1.00 & 1.00 \\
\hline & 1.00 & 1.00 & 1.00 & 1.00 & 1.00 & 1.00 & 1.00 & 1.00 & 1.00 & 1.00 & 1.00 & 1.00 & 1.00 & 1.00 & 1.00 & 1.00 & 1.00 & 1.00 \\
\hline \multirow[t]{5}{*}{$\lambda$} & -0.6572 & -0.9888 & -0.6572 & -0.9888 & -0.6572 & -0.9888 & -0.6572 & -0.9888 & -0.6572 & -0.9888 & -0.6572 & -0.9888 & -0.6572 & -0.9888 & -0.6572 & -0.9888 & -0.6572 & -0.9888 \\
\hline & 0.32 & 0.42 & 0.32 & 0.42 & 0.32 & 0.86 & 0.58 & 0.86 & 0.32 & 0.42 & 0.32 & 0.86 & 0.58 & 0.86 & 0.58 & 0.86 & 0.32 & 0.86 \\
\hline & 0.45 & 0.92 & 0.57 & 0.92 & 0.57 & 0.96 & 0.78 & 0.96 & 0.45 & 0.92 & 0.78 & 0.96 & 0.78 & 0.92 & 0.78 & 0.96 & 0.57 & 0.96 \\
\hline & 0.86 & 0.98 & 0.68 & 0.98 & 0.93 & 0.99 & 0.93 & 0.98 & 0.86 & 0.98 & 0.93 & 0.98 & 0.93 & 0.98 & 0.93 & 0.99 & 0.93 & 0.99 \\
\hline & 1.00 & 1.00 & 1.00 & 1.00 & 1.00 & 1.00 & 1.00 & 1.00 & 1.00 & 1.00 & 1.00 & 1.00 & 1.00 & 1.00 & 1.00 & 1.00 & 1.00 & 1.00 \\
\hline
\end{tabular}

A REAL PERSONNEL SELECTION PROBLEM USING THE GENERALIZED CHOQUET INTEGRAL METHODOLOGY 707 
Table 7: EvaluationUsing The Generalized Choquet Integral for $\alpha=1$

\begin{tabular}{|c|c|c|c|c|c|c|c|c|c|c|c|c|c|c|c|c|c|c|c|c|}
\hline \multirow{3}{*}{$\begin{array}{c}\text { Criteria } \\
\text { M1 }\end{array}$} & \multicolumn{2}{|c|}{ Importance of criteria } & \multicolumn{18}{|c|}{$\left[\mathrm{fi}^{-}, \mathrm{fi}^{+}\right]$} \\
\hline & & & \multicolumn{2}{|c|}{$\mathrm{A} 1$} & \multicolumn{2}{|c|}{ A2 } & \multicolumn{2}{|c|}{$\mathrm{A} 3$} & \multicolumn{2}{|c|}{ A4 } & \multicolumn{2}{|c|}{ A5 } & \multicolumn{3}{|c|}{ A6 } & \multicolumn{2}{|l|}{ A7 } & \multicolumn{2}{|l|}{ A8 } & A9 \\
\hline & & & 0.500 & 0.782 & 0.500 & 0.753 & 0.500 & 0.782 & 0.500 & 0.753 & 0.500 & 0.782 & 0.500 & 0.782 & 0.488 & 0.753 & 0.488 & 0.694 & 0.500 & 0.783 \\
\hline S11 & 0.98 & 0.98 & 0.49 & 0.6 & 0.5 & 0.61 & 0.49 & 0.6 & 0.49 & 0.6 & 0.49 & 0.6 & 0.49 & 0.6 & 0.49 & 0.6 & 0.49 & 0.6 & 0.5 & 0.61 \\
\hline $\mathrm{S} 12$ & 0.98 & 0.98 & 0.4 & 0.645 & 0.5 & 0.675 & 0.4 & 0.645 & 0.5 & 0.675 & 0.4 & 0.645 & 0.4 & 0.645 & 0.4 & 0.645 & 0.4 & 0.645 & 0.5 & 0.675 \\
\hline S13 & & 0.98 & 0.5 & 0.785 & 0.4 & 0.755 & 0.5 & 0.785 & 0.4 & 0.755 & 0.5 & 0.785 & 0.5 & 0.785 & 0.4 & 0.755 & 0.325 & 0.695 & 0.5 & 0.785 \\
\hline M2 & & & 0.483 & 0.755 & 0.500 & 0.784 & 0.483 & 0.755 & 0.483 & 0.755 & 0.498 & 0.784 & 0.423 & 0.755 & 0.389 & 0.695 & 0.389 & 0.695 & 0.483 & 0.755 \\
\hline S21 & 0.78 & 0.92 & 0.43 & 0.755 & 0.43 & 0.755 & 0.43 & 0.755 & 0.43 & 0.755 & 0.43 & 0.755 & 0.43 & 0.755 & 0.355 & 0.695 & 0.355 & 0.695 & 0.43 & 0.755 \\
\hline S22 & 0.78 & 0.92 & 0.5 & 0.675 & 0.5 & 0.675 & 0.5 & 0.675 & 0.5 & 0.675 & 0.4 & 0.645 & 0.4 & 0.645 & 0.4 & 0.645 & 0.4 & 0.645 & 0.5 & 0.675 \\
\hline S23 & 98 & 0.98 & 0.4 & 0.755 & 0.5 & 0.785 & 0.4 & 0.755 & 0.4 & 0.755 & 0.5 & 0.785 & 0.4 & 0.755 & 0.325 & 0.695 & 0.325 & 0.695 & 0.4 & 0.755 \\
\hline M3 & & & 0.396 & 0.835 & 0.419 & 0.847 & 0.468 & 0.870 & 0.411 & 0.837 & 0.407 & 0.836 & 0.388 & 0.788 & 0.369 & 0.787 & 0.337 & 0.725 & 0.468 & 0.870 \\
\hline S31 & 0.63 & 0.8 & 0.4 & 0.85 & 0.4 & 0.85 & 0.5 & 0.88 & 0.4 & 0.85 & 0.4 & 0.85 & 0.325 & 0.79 & 0.325 & 0.79 & 0.215 & 0.68 & 0.4 & 0.85 \\
\hline S32 & 0.63 & 0.8 & 0.325 & 0.79 & 0.4 & 0.85 & 0.4 & 0.85 & 0.325 & 0.79 & 0.325 & 0.79 & 0.325 & 0.79 & 0.325 & 0.79 & 0.215 & 0.68 & 0.5 & 0.88 \\
\hline S33 & 0.22 & 0.36 & 0.305 & 0.68 & 0.415 & 0.79 & 0.415 & 0.79 & 0.415 & 0.79 & 0.305 & 0.68 & 0.305 & 0.68 & 0.305 & 0.68 & 0.415 & 0.79 & 0.415 & 0.79 \\
\hline S34 & & 0.58 & 0.43 & 0.755 & 0.43 & 0.755 & 0.43 & 0.755 & 0.43 & 0.755 & 0.43 & 0.755 & 0.355 & 0.695 & 0.355 & 0.695 & 0.355 & 0.695 & 0.43 & 0.755 \\
\hline S35 & & & 0.32 & 0.63 & 0.415 & 0.74 & & 0.63 & & 0.63 & 0.32 & 0.63 & 0.415 & 0.74 & 0.32 & 0.63 & 0.32 & 0.63 & 0.415 & 0.74 \\
\hline S36 & & 0.58 & 0.32 & 0.63 & 0.415 & 0.74 & 0.415 & 0.74 & 0.415 & 0.74 & 0.415 & 0.74 & 0.415 & 0.74 & 0.415 & 0.74 & 0.32 & 0.63 & 0.32 & 0.63 \\
\hline M4 & & & 0.510 & 0.794 & 0.507 & 0.782 & 0.473 & 0.798 & 0.473 & 0.798 & 0.507 & 0.782 & 0.510 & 0.794 & 0.408 & 0.758 & 0.408 & 0.758 & 0.481 & 0.799 \\
\hline S41 & 0.22 & 0.36 & 0.305 & 0.68 & 0.415 & 0.79 & 0.415 & 0.79 & 0.415 & 0.79 & 0.415 & 0.79 & 0.305 & 0.68 & 0.305 & 0.68 & 0.305 & 0.68 & 0.415 & 0.79 \\
\hline S42 & 0.63 & 0.8 & 0.5 & 0.785 & 0.4 & 0.755 & 0.4 & 0.755 & 0.4 & 0.755 & 0.4 & 0.755 & 0.5 & 0.785 & 0.4 & 0.755 & 0.4 & 0.755 & 0.4 & 0.755 \\
\hline S43 & 0.98 & 0.98 & 0.5 & 0.675 & 0.5 & 0.675 & 0.4 & 0.645 & 0.4 & 0.645 & 0.5 & 0.675 & 0.5 & 0.675 & 0.4 & 0.645 & 0.4 & 0.645 & 0.4 & 0.645 \\
\hline S44 & 0.63 & 0.8 & 0.325 & 0.695 & 0.4 & 0.755 & 0.5 & 0.785 & 0.5 & 0.785 & 0.4 & 0.755 & 0.325 & 0.695 & 0.325 & 0.695 & 0.325 & 0.695 & 0.5 & 0.785 \\
\hline S45 & 0.78 & 0.92 & 0.43 & 0.755 & 0.355 & 0.695 & 0.355 & 0.695 & 0.355 & 0.695 & 0.355 & 0.695 & 0.43 & 0.755 & 0.355 & 0.695 & 0.355 & 0.695 & 0.43 & 0.755 \\
\hline S46 & 0.78 & 0.92 & 0.4 & 0.645 & 0.325 & 0.585 & 0.4 & 0.645 & 0.4 & 0.645 & 0.4 & 0.645 & 0.4 & 0.645 & 0.4 & 0.645 & 0.4 & 0.645 & 0.325 & 0.585 \\
\hline M5 & & & 0.410 & 0.836 & 0.422 & 0.839 & 0.416 & 0.831 & 0.459 & 0.852 & 0.410 & 0.836 & 0.402 & 0.828 & 0.381 & 0.823 & 0.377 & 0.818 & 0.396 & 0.783 \\
\hline S51 & 0.41 & 0.58 & 0.355 & 0.695 & 0.43 & 0.755 & 0.43 & 0.755 & 0.43 & 0.755 & 0.43 & 0.755 & 0.355 & 0.695 & 0.355 & 0.695 & 0.355 & 0.695 & 0.43 & 0.755 \\
\hline S52 & 0.63 & 0.8 & 0.4 & 0.85 & 0.4 & 0.85 & 0.4 & 0.85 & 0.5 & 0.88 & 0.4 & 0.85 & 0.4 & 0.85 & 0.4 & 0.85 & 0.4 & 0.85 & 0.325 & 0.79 \\
\hline S53 & 0.22 & 0.36 & 0.415 & 0.85 & 0.415 & 0.85 & 0.305 & 0.74 & 0.305 & 0.74 & 0.415 & 0.85 & 0.305 & 0.74 & 0.305 & 0.74 & 0.21 & 0.63 & 0.305 & 0.74 \\
\hline S54 & 0.41 & 0.58 & 0.43 & 0.755 & 0.43 & 0.755 & 0.43 & 0.755 & 0.355 & 0.695 & 0.355 & 0.695 & 0.43 & 0.755 & 0.355 & 0.695 & 0.355 & 0.695 & 0.43 & 0.755 \\
\hline
\end{tabular}

Business \& Management Studies: An International Journal Vol.:6 Issue:2 Year:2018 
Table 8: Fuzzy Weights $\mathrm{g}\left(\mathrm{A}_{(\mathrm{i})}\right)$ and $\lambda$ Values for $\alpha=1$

\begin{tabular}{|c|c|c|c|c|c|c|c|c|c|c|c|c|c|c|c|c|c|c|}
\hline & \multicolumn{18}{|c|}{$\left[g i=g\left(A_{(i)}\right), g i^{+}=g\left(A_{(i)}\right)\right]$} \\
\hline & \multicolumn{2}{|c|}{ A1 } & \multicolumn{2}{|c|}{ A2 } & \multicolumn{2}{|c|}{ A3 } & \multicolumn{2}{|c|}{ A4 } & \multicolumn{2}{|c|}{ A5 } & \multicolumn{2}{|c|}{ A6 } & \multicolumn{2}{|c|}{ A7 } & \multicolumn{2}{|c|}{ A8 } & \multicolumn{2}{|c|}{ A9 } \\
\hline$\lambda$ & -0.9997 & -1 & -0.9997 & -1 & -0.9997 & -1 & -0.9997 & -1 & -0.9997 & -1 & -0.9997 & -1 & -0.9997 & -1 & -0.9997 & -1 & -0.9997 & -1 \\
\hline & 0.93 & 1.00 & 0.93 & 1.00 & 0.93 & 1.00 & 0.93 & 1.00 & 0.93 & 1.00 & 0.93 & 1.00 & 0.93 & 1.00 & 0.93 & 1.00 & 0.93 & 1.00 \\
\hline & 1.00 & 1.00 & 1.00 & 1.00 & 1.00 & 1.00 & 1.00 & 1.00 & 1.00 & 1.00 & 1.00 & 1.00 & 1.00 & 1.00 & 1.00 & 1.00 & 1.00 & 1.00 \\
\hline & 1.00 & 1.00 & 1.00 & 1.00 & 1.00 & 1.00 & 1.00 & 1.00 & 1.00 & 1.00 & 1.00 & 1.00 & 1.00 & 1.00 & 1.00 & 1.00 & 1.00 & 1.00 \\
\hline \multirow[t]{4}{*}{$\lambda$} & -0.9939 & -1 & -0.9939 & -1 & -0.9939 & -1 & -0.9939 & -1 & -0.9939 & -1 & -0.9939 & -1 & -0.9939 & -1 & -0.9939 & -1 & -0.9939 & -1 \\
\hline & 0.72 & 0.97 & 0.72 & 1.00 & 0.72 & 0.97 & 0.72 & 0.97 & 0.93 & 1.00 & 0.72 & 0.97 & 0.72 & 0.97 & 0.72 & 0.97 & 0.72 & 0.97 \\
\hline & 0.92 & 1.00 & 0.98 & 1.00 & 0.92 & 1.00 & 0.92 & 1.00 & 0.98 & 1.00 & 0.92 & 1.00 & 0.92 & 1.00 & 0.92 & 1.00 & 0.92 & 1.00 \\
\hline & 1.00 & 1.00 & 1.00 & 1.00 & 1.00 & 1.00 & 1.00 & 1.00 & 1.00 & 1.00 & 1.00 & 1.00 & 1.00 & 1.00 & 1.00 & 1.00 & 1.00 & 1.00 \\
\hline \multirow[t]{7}{*}{$\lambda$} & -0.9408 & -0.9995 & -0.9408 & -0.9995 & -0.9408 & -0.9995 & -0.9408 & -0.9995 & -0.9408 & -0.9995 & -0.9408 & -0.9995 & -0.9408 & -0.9995 & -0.9408 & -0.9995 & -0.9408 & -0.9995 \\
\hline & 0.32 & 0.86 & 0.32 & 0.86 & 0.58 & 0.86 & 0.32 & 0.86 & 0.32 & 0.86 & 0.32 & 0.86 & 0.32 & 0.86 & 0.17 & 0.42 & 0.58 & 0.86 \\
\hline & 0.73 & 0.98 & 0.44 & 0.98 & 0.73 & 0.98 & 0.44 & 0.98 & 0.73 & 0.98 & 0.54 & 0.98 & 0.54 & 0.98 & 0.44 & 0.80 & 0.73 & 0.98 \\
\hline & 0.91 & 0.99 & 0.78 & 0.99 & 0.78 & 0.99 & 0.78 & 0.99 & 0.83 & 0.99 & 0.70 & 0.99 & 0.83 & 0.99 & 0.63 & 0.97 & 0.78 & 0.99 \\
\hline & 0.96 & 1.00 & 0.93 & 1.00 & 0.93 & 1.00 & 0.86 & 1.00 & 0.96 & 1.00 & 0.90 & 1.00 & 0.96 & 1.00 & 0.76 & 1.00 & 0.93 & 1.00 \\
\hline & 0.99 & 1.00 & 0.97 & 1.00 & 0.97 & 1.00 & 0.97 & 1.00 & 0.99 & 1.00 & 0.99 & 1.00 & 0.99 & 1.00 & 0.92 & 1.00 & 0.97 & 1.00 \\
\hline & 1.00 & 1.00 & 1.00 & 1.00 & 1.00 & 1.00 & 1.00 & 1.00 & 1.00 & 1.00 & 1.00 & 1.00 & 1.00 & 1.00 & 1.00 & 1.00 & 1.00 & 1.00 \\
\hline \multirow[t]{7}{*}{$\lambda$} & -0.9992 & -1 & -0.9992 & -1 & -0.9992 & -1 & -0.9992 & -1 & -0.9992 & -1 & -0.9992 & -1 & -0.9992 & -1 & -0.9992 & -1 & -0.9992 & -1 \\
\hline & 0.58 & 0.86 & 0.93 & 0.42 & 0.58 & 0.42 & 0.58 & 0.42 & 0.93 & 0.42 & 0.58 & 0.86 & 0.58 & 0.86 & 0.58 & 0.86 & 0.58 & 0.42 \\
\hline & 0.97 & 1.00 & 0.94 & 0.92 & 0.65 & 0.92 & 0.65 & 0.92 & 0.94 & 0.92 & 0.97 & 1.00 & 0.88 & 0.98 & 0.88 & 0.98 & 0.88 & 0.92 \\
\hline & 0.99 & 1.00 & 0.98 & 0.99 & 0.85 & 0.99 & 0.85 & 0.99 & 0.98 & 0.99 & 0.99 & 1.00 & 0.99 & 1.00 & 0.99 & 1.00 & 0.90 & 0.99 \\
\hline & 1.00 & 1.00 & 0.99 & 1.00 & 0.96 & 1.00 & 0.96 & 1.00 & 0.99 & 1.00 & 1.00 & 1.00 & 1.00 & 1.00 & 1.00 & 1.00 & 0.96 & 1.00 \\
\hline & 1.00 & 1.00 & 1.00 & 1.00 & 1.00 & 1.00 & 1.00 & 1.00 & 1.00 & 1.00 & 1.00 & 1.00 & 1.00 & 1.00 & 1.00 & 1.00 & 1.00 & 1.00 \\
\hline & 1.00 & 1.00 & 1.00 & 1.00 & 1.00 & 1.00 & 1.00 & 1.00 & 1.00 & 1.00 & 1.00 & 1.00 & 1.00 & 1.00 & 1.00 & 1.00 & 1.00 & 1.00 \\
\hline \multirow[t]{5}{*}{$\lambda$} & -0.6572 & -0.9888 & -0.6572 & -0.9888 & -0.6572 & -0.9888 & -0.6572 & -0.9888 & -0.6572 & -0.9888 & -0.6572 & -0.9888 & -0.6572 & -0.9888 & -0.6572 & -0.9888 & -0.6572 & -0.9888 \\
\hline & 0.32 & 0.42 & 0.32 & 0.42 & 0.32 & 0.86 & 0.58 & 0.86 & 0.32 & 0.42 & 0.32 & 0.86 & 0.58 & 0.86 & 0.58 & 0.86 & 0.32 & 0.86 \\
\hline & 0.45 & 0.92 & 0.57 & 0.92 & 0.57 & 0.96 & 0.78 & 0.96 & 0.45 & 0.92 & 0.78 & 0.96 & 0.78 & 0.92 & 0.78 & 0.96 & 0.57 & 0.96 \\
\hline & 0.86 & 0.98 & 0.68 & 0.98 & 0.93 & 0.99 & 0.93 & 0.98 & 0.86 & 0.98 & 0.93 & 0.98 & 0.93 & 0.98 & 0.93 & 0.99 & 0.93 & 0.99 \\
\hline & 1.00 & 1.00 & 1.00 & 1.00 & 1.00 & 1.00 & 1.00 & 1.00 & 1.00 & 1.00 & 1.00 & 1.00 & 1.00 & 1.00 & 1.00 & 1.00 & 1.00 & 1.00 \\
\hline
\end{tabular}


Table 9: Overall Values of Alternatives Using Choquet Integral

\begin{tabular}{|c|c|c|c|c|c|c|c|c|c|c|c|c|c|c|c|c|c|c|c|c|c|c|c|c|}
\hline \multirow[t]{2}{*}{ Criteria } & \multicolumn{4}{|c|}{ A1 } & \multicolumn{4}{|c|}{ A2 } & \multicolumn{4}{|c|}{ A3 } & \multicolumn{4}{|c|}{ A4 } & \multicolumn{4}{|c|}{ A5 } & \multicolumn{4}{|c|}{ A6 } \\
\hline & 0.464 & 0.507 & 0.845 & 893 & 494 & 0.505 & 0.840 & .896 & 462 & 0.499 & 0.860 & & & 0.499 & 0.842 & & 0.464 & 0.505 & 0.845 & 0.893 & 0.462 & 0.483 & 0.772 & 0.872 \\
\hline M1 & 464 & 800 & & & & & & & & & & & & & & & & & & & & & 0.782 & \\
\hline S11 & 465 & 490 & 600 & 640 & 500 & & 010 & & & & & & & & & & | 0.403 & & 0.600 & 0.640 & 0.465 & 0.490 & .600 & .640 \\
\hline S12 & 360 & 400 & 45 & 695 & 465 & 500 & .675 & 10 & 360 & .400 & & & 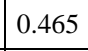 & 0.000 & T. & & 0.360 & 0.400 & .045 & 0.099 & 0.360 & 0.400 & .645 & 695 \\
\hline $\mathrm{S} 13$ & 465 & 500 & 785 & 840 & 360 & 400 & 55 & 25 & .465 & .500 & 785 & & 0.360 & 0.400 & 755 & 825 & 465 & 0.500 & .785 & 0.840 & .465 & 0.500 & .785 & 840 \\
\hline M2 & 439 & 483 & 755 & 825 & .464 & .500 & .784 & 0.840 & .439 & 0.483 & 0.755 & 8.825 & 0.439 & 0.483 & 0.755 & 0.825 & 0.458 & 0.498 & 0.784 & 0.840 & 0.371 & 0.423 & .755 & 825 \\
\hline $\mathrm{S} 21$ & 375 & 430 & .755 & 825 & 375 & .430 & .755 & 1825 & .375 & 0.430 & 0.755 & 0.825 & 0.375 & 0.430 & 0.755 & 0.825 & 0.375 & 0.430 & 0.755 & 0.825 & 0.375 & 0.430 & .755 & .825 \\
\hline $\mathrm{S} 22$ & 0.465 & 0.500 & 0.675 & 0.710 & 0.465 & 0.500 & 0.675 & 0.710 & 0.465 & 0500 & 0.675 & 0710 & 0.465 & 0.500 & 0.675 & 0.710 & 0.360 & 0.400 & 0.645 & 0.695 & 0.360 & 0.400 & 0.645 & 0.695 \\
\hline $\mathrm{S} 23$ & 0 & 400 & 0755 & 0825 & 0.465 & 0500 & 0785 & 0.840 & 0360 & 0,400 & 0755 & 0825 & 0360 & 0.400 & 0755 & 0.825 & 0.465 & 0500 & 0785 & 0.840 & 0.360 & 0.400 & 0.755 & 0.825 \\
\hline M3 & 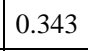 & . & 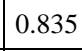 & 801 & 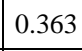 & . & 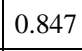 & 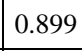 & 4 & 0.100 & . & 0.011 & 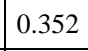 & 0.11 & ד & D. & D. & 政 & 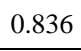 & 0.892 & 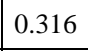 & 0.000 & 0.788 & 0.844 \\
\hline S31 & 500 & $\$ 00$ & 0 & 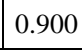 & 360 & 400 & .050 & 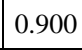 & .465 & 0.500 & 0.00 & & 0.000 & 0.00 & 0.000 & & 0.000 & 0.00 & 0.000 & 0.300 & 0.230 & 0.025 & 0.790 & 0.845 \\
\hline S32 & 290 & 320 & 130 & $0+J$ & 360 & 4.400 & .000 & 00 & .360 & 0.400 & | & & 0.230 & | & $\mid 0.170$ & | & 0.290 & | & 0.190 & 0.845 & 0.290 & 0.325 & 0.790 & 0.845 \\
\hline S33 & 230 & 305 & 680 & .740 & .360 & .415 & .790 & 0.845 & .360 & 0.415 & 0.790 & 0.845 & 0.360 & 0.415 & 0.790 & $\mid 0.845$ & 0.230 & 0.305 & 0.680 & 0.740 & 0.230 & 0.305 & 0.680 & 0.740 \\
\hline S34 & 375 & 430 & 755 & 825 & 375 & 430 & .755 & .825 & 0.375 & 0.430 & 0.755 & 0.825 & 0.375 & 0.430 & 0.755 & 0.825 & 0.375 & 0.430 & 0.755 & 0.825 & 0.305 & 0.355 & 0.695 & 0.770 \\
\hline S35 & 260 & 320 & 630 & 690 & .335 & 415 & 0.740 & 0.805 & 0.260 & 0.320 & 0.630 & 0.690 & 0.260 & 0.320 & 0.630 & $\mid 0.690$ & 0.260 & 0.320 & 0.630 & 0.690 & 0.335 & 0.415 & 0.740 & 0.805 \\
\hline S36 & 0.260 & 0.320 & 0.630 & 0.690 & 0.335 & 0.415 & 0.740 & 0.805 & 0.335 & 0.415 & 0.740 & 0.805 & 0.335 & 0.415 & 0.740 & 0.805 & 0.335 & 0.415 & 0.740 & 0.805 & 0.335 & 0.415 & 0.740 & 0.805 \\
\hline M4 & 0.462 & 0.499 & 0778 & 838 & 0.457 & 0.498 & 0.766 & 0833 & 0.421 & 0.464 & 0.781 & 0.840 & 0.421 & 0.464 & 0.781 & 0.840 & 0.458 & 0.498 & 0.766 & 0.833 & 0.462 & 0.499 & 0.778 & 0.838 \\
\hline S41 & 0.230 & 0305 & 0680 & 0740 & 0.360 & 0.415 & 0.790 & 0.845 & 0.360 & 0.415 & 0.790 & 0.845 & 0.360 & 0.415 & 0.790 & 0.845 & 0.360 & 0.415 & 0.790 & 0.845 & 0.230 & 0.305 & 0.680 & 0.740 \\
\hline $\mathrm{S} 42$ & 0.465 & 0500 & 078 & O & 036 & 0400 & 0755 & & 0.360 & 0.400 & 0.755 & & 0.360 & 0.400 & & 0.825 & 0.360 & 0.400 & 0755 & 0.825 & 0465 & 0.500 & 0.785 & 0.840 \\
\hline S43 & & & & & & & & & & 0.400 & & & & & & & & 0.500 & & 0.710 & $0.40 J$ & 0.500 & 0.675 & 0.710 \\
\hline S44 & 0.290 & . & 0.078 & 0.170 & 0.500 & 0.400 & 0.755 & & 0.465 & 0.500 & 0.785 & & & 0.500 & & & 0.360 & 0.400 & 0.755 & 0.825 & 0.290 & 0.325 & 0.695 & 0.770 \\
\hline S45 & 375 & 430 & 0.135 & 0.025 & 0.305 & 0.355 & 0.695 & 0.770 & 0.305 & 0.355 & 0.695 & 0.770 & 0.305 & 0.355 & 0.695 & $\mid 0.770$ & 0.305 & 0.355 & 0.695 & 0.770 & 0.375 & 0.430 & 0.755 & 0.825 \\
\hline S46 & 0.360 & 0.400 & 0.645 & 0.695 & 0.290 & 0.325 & 0.585 & 0.640 & 0.360 & 0.400 & 0.645 & 0.695 & 0.360 & 0.400 & 0.645 & 0.695 & $\mid 0.360$ & 0.400 & 0.645 & 0.695 & 0.360 & 0.400 & 0.645 & 0.695 \\
\hline M5 & 0.357 & 0.410 & 0.836 & 0.897 & 0.369 & 0.422 & 0.839 & 0.898 & 0.360 & 0.416 & 0.831 & 0.889 & \begin{tabular}{|l|l|}
0.407 \\
\end{tabular} & \begin{tabular}{|l|l|}
0.459 \\
\end{tabular} & 0.852 & 0.901 & 0.357 & 0.410 & 0.836 & 0.897 & 0.348 & 0.402 & 0.828 & 0.888 \\
\hline S51 & 0.305 & 355 & 695 & 70 & 375 & 0.430 & 0.7 & & 0.3 & 0.430 & 0.755 & & 0.375 & 0.430 & 0.755 & & 0.375 & 0.430 & 0.755 & 0.825 & 0.305 & 0.355 & 0.695 & 0.770 \\
\hline S52 & 0.360 & 0.400 & & & .360 & 0.400 & & & & 0.400 & & & & & & & & 0.40 & & 900 & 360 & 0.400 & 850 & 900 \\
\hline S53 & 0.360 & 0.415 & 0.850 & & & & 0.850 & & & 0.305 & 0.740 & & & & & & & & & 0.910 & 0.230 & 0.305 & 0.740 & 0.805 \\
\hline S54 & 0.375 & 0.430 & 0.755 & 0.825 & 0.375 & 0.430 & 0.755 & 0.825 & 0.375 & 0.430 & 0.755 & 0.825 & 0.305 & 0.355 & 0.695 & $\mid 0.770$ & 0.305 & 0.355 & 0.695 & 0.770 & 0.375 & 0.430 & 0.755 & 0.020 \\
\hline
\end{tabular}


Table 9: Overall Values of Alternatives Using Choquet Integral (continue)

\begin{tabular}{|c|c|c|c|c|c|c|c|c|c|c|c|c|c|c|c|c|c|c|c|c|c|c|c|c|}
\hline & \multicolumn{4}{|c|}{ At } & \multicolumn{4}{|c|}{ A8 } & \multicolumn{4}{|c|}{ A9 } & \multicolumn{4}{|c|}{ A10 } & \multicolumn{4}{|c|}{ A11 } & \multicolumn{4}{|c|}{ A12 } \\
\hline & & & & 869 & & & & & & & & & & & & & & & & & & & .792 & \\
\hline & & & & & & & & & & & & & & & & & & & & & & & & \\
\hline & & & & & & & & & & & & & & & & & & & & & & & & \\
\hline $\mathrm{S} 12$ & & & & & & & & & & & & & & & & & & & & & & & & \\
\hline $\mathrm{S} 13$ & & & & & & & & & & & & & & & & & & & & & & & & 0.78 \\
\hline ר & & & & & & & & & & & & & & & & & & & & & & & & . \\
\hline S21 & & & & & & & & & & & & & & & & & & & & & & & & \\
\hline S22 & & 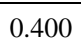 & 8 & 0 & & & & . & .465 & & & & & 0.400 & & & & 0.400 & & & & & & \\
\hline$S 23$ & & 325 & 605 & 770 & 290 & & & 770 & 0.360 & & & & & 325 & & & & 325 & & & & & & \\
\hline 93 & & & 787 & 844 & & & & 705 & 0.419 & & & & & & & & & & & & & & & \\
\hline $\mathrm{S} 31$ & & & 700 & 815 & & & & 0740 & 0360 & & & & & 0.400 & & & & 0.325 & 0700 & & & & & \\
\hline $\mathrm{S} 32$ & & 5 & 790 & 845 & & & & 0 & 5 & & & & & & & & & & & & & & & \\
\hline $\mathrm{S}$ & 230 & 305 & 680 & 740 & 360 & 415 & 700 & 0.845 & 0.360 & 415 & 700 & 815 & 230 & 305 & 680 & 710 & 232 & 0.305 & 680 & 740 & .360 & 0415 & .790 & 845 \\
\hline S34 & 305 & 355 & 695 & .770 & 305 & 355 & 695 & 0.770 & 0.375 & 0.430 & 755 & 875 & 0305 & 0.355 & 0.695 & 770 & 0.305 & 0.355 & 0.695 & 0.770 & 0.305 & 0.355 & 0.695 & 0770 \\
\hline S35 & 260 & 320 & 630 & 690 & 260 & 320 & 630 & .690 & 0.335 & 0.415 & 740 & 805 & 260 & 0.320 & 0,630 & 690 & 0260 & 0.320 & 0.630 & 0.690 & 0.260 & 0.320 & 0.630 & 0.690 \\
\hline S36 & 35 & 115 & 740 & 805 & 260 & 320 & 630 & 0.690 & 0.260 & 0.320 & 630 & 6000 & 0335 & 0.415 & 0.740 & 805 & 027 & 0.415 & 0.740 & 0.805 & .260 & 0.3 & ).630 & 0.690 \\
\hline M4 & 360 & 400 & 743 & 817 & 360 & 400 & 743 & 0.817 & 0.425 & 0.472 & 783 & 0.841 & 0.371 & 0.424 & 0.767 & 0.833 & 0.360 & 0.400 & 0.743 & 0.817 & .360 & 0.400 & .743 & 0.817 \\
\hline S41 & 230 & 305 & 680 & 740 & 230 & 305 & 680 & 0.740 & 0.360 & 0.415 & 790 & 0.845 & 0.360 & 0.415 & 0.790 & 0.845 & 0.230 & 0.305 & 0.680 & 0.740 & 0.230 & 0.305 & ).680 & 0.74 \\
\hline S42 & 360 & 400 & .755 & 825 & 360 & 400 & 0.755 & 0.825 & 0.360 & 0.400 & 0.755 & 0.825 & 0.360 & 0.400 & 0.755 & 0.825 & 0.360 & 0.400 & 0.755 & 0.825 & 0.360 & 0.400 & 0.755 & 0.825 \\
\hline S43 & 360 & 400 & 645 & 695 & 360 & 400 & 645 & 0.695 & 0.360 & 0.400 & 645 & 0.695 & 0.360 & 0.400 & 0.645 & 0.695 & 0.360 & 0.400 & 0.645 & 0.695 & 0.360 & 0.400 & .645 & 60 \\
\hline S44 & 290 & 325 & 695 & 770 & 290 & 325 & 0.695 & 0.770 & 0.465 & 500 & 785 & 0.840 & 290 & 325 & 0.695 & 0.770 & 20 & 0.325 & 0.695 & 0.770 & .290 & 325 & 695 & 77 \\
\hline 45 & 05 & 5 & & 170 & & & & 70 & & & & & & & & & & & 595 & 170 & 305 & & & 0.77 \\
\hline$S$ & 60 & 0 & & & & & & & & & & & & & & & & & & & 360 & 400 & 645 & 595 \\
\hline M5 & 332 & 881 & 23 & 884 & & & & 81 & & 396 & & & & & & & & & 23 & & 327 & 377 & 818 & 381 \\
\hline S51 & 305 & 355 & 5 & 0.770 & 305 & 355 & 95 & 0.770 & 75 & +30 & & & & & & & 0.305 & 355 & 695 & & 0.305 & 55 & .695 & 0.770 \\
\hline S52 & 660 & 100 & 50 & 900 & & & & 0.900 & 90 & 0.325 & 790 & 0.845 & 0.30 & 0.400 & 0.850 & 0.30 & 0.360 & 0.400 & 0.850 & 0.900 & 0.360 & 0.400 & .850 & 0.900 \\
\hline S53 & 0.230 & 0.305 & 0.740 & 0.805 & 0.155 & 0.210 & 0.630 & 0.690 & 0.230 & 0.305 & 0.740 & 0.805 & 0.230 & 0.305 & 0.740 & 0.005 & 0.230 & 0.305 & 0.740 & 0.805 & 0.155 & 0.210 & 0.630 & 0.690 \\
\hline S54 & 0.305 & 0.355 & 0.695 & 0.770 & 0.305 & 0.355 & 0.695 & 0.770 & 0.375 & 0.430 & 0.755 & 0.825 & 0.375 & 0.430 & 0.755 & 0.825 & 0.305 & 0.355 & 0.695 & 0.770 & 0.305 & 0.355 & 0.695 & 0.770 \\
\hline
\end{tabular}




\section{RESULTS}

In Table 9, using the calculation for Choquet integral in Application section, the performance of alternative personnel are obtained. In addition, the defuzzified overall values of alternatives are shown in Table 10. For example, the value (0.677) of "A1 and overall personnel alternative value" is obtained in that way (11):

$$
\frac{0.464+0.507+0.845+0.893}{4}=0.677
$$

The defuzzified overall values of alternatives are calculated by generalized Choquet Integral as follows: $0.677,0.684,0.682,0.675,0.677,0.647,0.663,0.647,0.688,0.668,0.663$, $0.647,0.602,0.584,0.573,0.568,0.508$.

From the results shown in Table 10, the personnel ranking is obtained as "A9 $>\mathrm{A} 2>\mathrm{A} 3>\mathrm{A} 1=\mathrm{A} 5>\mathrm{A} 4>\mathrm{A} 10>\mathrm{A} 7=\mathrm{A} 11>\mathrm{A} 6=\mathrm{A} 8=\mathrm{A} 12>\mathrm{A} 13>\mathrm{A} 14>\mathrm{A} 15>\mathrm{A} 16>\mathrm{A} 17 "$. Given these results, it is fair to say that selecting Personnel "A9" is the most reasonable outcome, followed by the others. However, the best alternative "A9" has the largest weights for activity (M1) and education (M3) main criteria; the other alternatives "A1" and "A6" have the largest weight for internal factors (M4) main criteria; "A2" is for fee (M2) main criteria; "A3" is for education (M3) main criteria; and "A4" is for business factors (M5) main criteria. 
Table 10: Defuzzified Values of Alternatives Using Choquet Integral

\begin{tabular}{|c|c|c|c|c|c|c|c|c|c|c|c|c|c|c|c|c|c|}
\hline Criteria & A1 & A2 & A3 & A4 & A5 & A6 & A7 & A8 & A9 & A10 & A11 & A12 & A13 & A14 & A15 & A16 & A17 \\
\hline & 0.677 & 0.684 & 0.682 & 0.675 & 0.677 & 0.647 & 0.663 & 0.647 & 0.688* & 0.668 & 0.663 & 0.647 & 0.602 & 0.584 & 0.573 & 0.568 & 0.508 \\
\hline M1 & 0.647 & 0.644 & 0.647 & 0.636 & 0.647 & 0.647 & 0.631 & 0.602 & $0.655^{*}$ & 0.631 & 0.631 & 0.602 & 0.524 & 0.524 & 0.498 & 0.498 & 0.498 \\
\hline S11 & 0.549 & $0.563^{*}$ & 0.549 & 0.549 & 0.549 & 0.549 & 0.549 & 0.549 & $0.563^{*}$ & 0.549 & 0.549 & 0.549 & 0.486 & 0.486 & 0.486 & 0.486 & 0.486 \\
\hline S12 & 0.525 & $0.588^{*}$ & 0.525 & $0.588^{*}$ & 0.525 & 0.525 & 0.525 & 0.525 & $0.588^{*}$ & 0.525 & 0.525 & 0.525 & 0.525 & 0.525 & 0.460 & 0.460 & 0.460 \\
\hline $\mathrm{S} 13$ & $0.648^{*}$ & 0.585 & $0.648^{*}$ & 0.585 & $0.648^{*}$ & $0.648 *$ & 0.585 & 0.520 & $0.648 *$ & 0.585 & 0.585 & 0.520 & 0.406 & 0.406 & 0.406 & 0.406 & 0.406 \\
\hline M2 & 0.625 & $0.647^{*}$ & 0.625 & 0.625 & 0.645 & 0.593 & 0.549 & 0.549 & 0.625 & 0.590 & 0.549 & 0.549 & 0.546 & 0.519 & 0.453 & 0.519 & 0.453 \\
\hline S21 & $596 *$ & $0.596^{*}$ & $0.596^{*}$ & $0.596^{*}$ & $0.596^{*}$ & $0.596^{*}$ & 0.531 & 0.531 & $0.596 *$ & $0.596^{*}$ & 0.531 & 0.531 & 0.418 & 0.418 & 0.418 & 0.418 & 0.418 \\
\hline S22 & $0.588^{*}$ & 0.588* & $0.588^{*}$ & $0.588^{*}$ & 0.525 & 0.525 & 0.525 & 0.525 & $0.588 *$ & 0.525 & 0.525 & 0.525 & 0.525 & 0.460 & 0.460 & 0.460 & 0.460 \\
\hline S23 & 0.585 & $0.648^{*}$ & 0.585 & 0.585 & $0.648^{*}$ & 0.585 & 0.520 & 0.520 & 0.585 & 0.520 & 0.520 & 0.520 & 0.520 & 0.520 & 0.406 & 0.520 & 0.406 \\
\hline M3 & 0.616 & 0.632 & $0.667 *$ & 0.623 & 0.621 & 0.584 & 0.577 & 0.530 & $0.667 *$ & 0.614 & 0.577 & 0.530 & 0.523 & 0.488 & 0.532 & 0.483 & 0.407 \\
\hline S31 & 0.628 & 0.628 & $0.690^{*}$ & 0.628 & 0.628 & 0.563 & 0.563 & 0.449 & 0.628 & 0.628 & 0.563 & 0.449 & 0.449 & 0.449 & 0.563 & 0.449 & 0.350 \\
\hline S32 & 0.563 & 0.628 & 0.628 & 0.563 & 0.563 & 0.563 & 0.563 & 0.449 & $0.690 *$ & 0.563 & 0.563 & 0.449 & 0.350 & 0.350 & 0.350 & 0.350 & 0.350 \\
\hline S33 & 0.489 & $0.603 *$ & $0.603^{*}$ & $0.603^{*}$ & 0.489 & 0.489 & 0.489 & $0.603^{*}$ & $0.603^{*}$ & 0.489 & 0.489 & 0.603 & 0.603 & 0.489 & 0.390 & 0.390 & 0.390 \\
\hline S34 & $0.596^{*}$ & $0.596^{*}$ & $0.596^{*}$ & $0.596^{*}$ & $0.596^{*}$ & 0.531 & 0.531 & 0.531 & $0.596^{*}$ & 0.531 & 0.531 & 0.531 & 0.531 & 0.531 & 0.418 & 0.531 & 0.418 \\
\hline S35 & 0.475 & $0.574 *$ & 0.475 & 0.475 & 0.475 & $0.574 *$ & 0.475 & 0.475 & $0.574 *$ & 0.475 & 0.475 & 0.475 & 0.398 & 0.398 & 0.475 & 0.398 & 0.398 \\
\hline S36 & 0.475 & $0.574 *$ & 0.574* & 0.574* & $0.574 *$ & $0.574 *$ & $0.574 *$ & 0.475 & 0.475 & 0.574* & 0.574* & 0.475 & 0.475 & 0.398 & 0.398 & 0.398 & 0.398 \\
\hline M4 & 0.644* & 0.638 & 0.627 & 0.627 & 0.639 & $0.644 *$ & 0.580 & 0.580 & 0.630 & 0.599 & 0.580 & 0.580 & 0.576 & 0.559 & 0.525 & 0.528 & 0.466 \\
\hline S41 & 0.489 & $0.603^{*}$ & $0.603^{*}$ & $0.603^{*}$ & $0.603^{*}$ & 0.489 & 0.489 & 0.489 & $0.603 *$ & $0.603^{*}$ & 0.489 & 0.489 & 0.489 & 0.489 & 0.390 & 0.390 & 0.390 \\
\hline S42 & $0.648 *$ & 0.585 & 0.585 & 0.585 & 0.585 & $0.648^{*}$ & 0.585 & 0.585 & 0.585 & 0.585 & 0.585 & 0.585 & 0.585 & 0.585 & 0.406 & 0.520 & 0.406 \\
\hline S43 & $0.588^{*}$ & $0.588^{*}$ & 0.525 & 0.525 & $0.588^{*}$ & $0.588^{*}$ & 0.525 & 0.525 & 0.525 & 0.525 & 0.525 & 0.525 & 0.460 & 0.460 & 0.460 & 0.460 & 0.460 \\
\hline S44 & 0.520 & 0.585 & $0.648 *$ & $0.648^{*}$ & 0.585 & 0.520 & 0.520 & 0.520 & $0.648 *$ & 0.520 & 0.520 & 0.520 & 0.520 & 0.406 & 0.406 & 0.406 & 0.406 \\
\hline S45 & $0.596^{*}$ & 0.531 & 0.531 & 0.531 & 0.531 & $0.596^{*}$ & 0.531 & 0.531 & $0.596^{*}$ & $0.596^{*}$ & 0.531 & 0.531 & 0.418 & 0.418 & 0.531 & 0.531 & 0.418 \\
\hline S46 & $0.525^{*}$ & 0.460 & $0.525^{*}$ & $0.525^{*}$ & $0.525^{*}$ & $0.525^{*}$ & $0.525^{*}$ & $0.525^{*}$ & 0.460 & $0.525^{*}$ & $0.525^{*}$ & $0.525^{*}$ & $0.525^{*}$ & 0.460 & 0.460 & 0.460 & 0.460 \\
\hline M5 & 0.625 & 0.632 & 0.624 & $0.655^{*}$ & 0.625 & 0.617 & 0.605 & 0.601 & 0.589 & 0.617 & 0.605 & 0.601 & 0.598 & 0.556 & 0.446 & 0.524 & 0.408 \\
\hline S51 & 0.531 & $0.596 *$ & $0.596^{*}$ & $0.596^{*}$ & $0.596^{*}$ & 0.531 & 0.531 & 0.531 & $0.596^{*}$ & 0.531 & 0.531 & 0.531 & 0.531 & 0.531 & 0.418 & 0.418 & 0.418 \\
\hline S52 & 0.628 & 0.628 & 0.628 & $0.690^{*}$ & 0.628 & 0.628 & 0.628 & 0.628 & 0.563 & 0.628 & 0.628 & 0.628 & 0.628 & 0.563 & 0.449 & 0.563 & 0.350 \\
\hline S53 & $0.634 *$ & $0.634 *$ & 0.520 & 0.520 & $0.634 *$ & 0.520 & 0.520 & 0.421 & 0.520 & 0.520 & 0.520 & 0.421 & 0.344 & 0.344 & 0.344 & 0.344 & 0.421 \\
\hline S54 & $0.596^{*}$ & $0.596^{*}$ & $0.596^{*}$ & 0.531 & 0.531 & $0.596^{*}$ & 0.531 & 0.531 & $0.596^{*}$ & $0.596^{*}$ & 0.531 & 0.531 & 0.531 & 0.531 & 0.418 & 0.418 & 0.418 \\
\hline
\end{tabular}

* The best alternative for criteria 


\section{CONCLUSION}

In the previous study (Ozdemir et al., 2017), a multi-criteria decision making technique, Consistent Fuzzy Preference Relations (CFPR) method was used for the evaluation of personnel selection criteria. However, this technique can only prioritize personnel selection criteria and it cannot select the best alternative. For this reason, in this study another MCDM technique, the generalized Choquet integral method using trapezoidal fuzzy numbers, is used for prioritization of personnel.

A Choquet integral methodology considers interactivity among main criteria and subcriteria. Using trapezoidal fuzzy numbers and the range computations of Choquet integral can enable better results for daily usage. In addition, the proposed methodology has an ability of evaluating personnel selection information from internal and external environments. The main advantage of the proposed problem is to indicate the impact of this interactivity using trapezoidal fuzzy numbers. The main contribution of this paper is to determine the interdependency and the environmental uncertainties while prioritizing personnel alternatives.

Personnel selection is usually done manually and subjectively in firms. For this reason in this study we aimed to determine the criteria for personnel selection, and aimed to use Choquet integral methodology for decision making. The criteria determined in this study were grouped into main criteria and subcriteria. According to the experts' view, that consist of from academicians and managers, 5 main criteria and 22 subcriteria were determined. All of these criteria cover the efficient work, fee, education, internal and external factors to be taken into consideration for a promoted personnel.

As a result of evaluation process, this MCDM method, generalized Choquet integral, has determined the most suitable personnel as A9. The ranking of the other alternatives are $\mathrm{A} 2>\mathrm{A} 3>\mathrm{A} 1=\mathrm{A} 5>\mathrm{A} 4$. Also, the alternative "A9" has the largest weights for activity (M1) and education (M3) main criteria, at the same time for S11, S12, S13, S21, S22, S32, S33, S34, S35, S41, S44, S45, S51, and S54 subcriteria among all alternative personnel.

As regards future research, intelligent software to calculate solutions automatically could be developed. By this way, the Choquet integral methodology could be used easily by the firms. Also, this methodology can be applied to many other decision making problems for human resources besides the promotion. 


\section{REFERENCES}

Afshari, A. R., Mojahed, M., Yusuff, R. M., Hong, T. S. \& Ismail, M. Y. (2010). Personnel selection using ELECTRE. Journal of Applied Sciences, 10, 3068-3075.

Baležentis, A., Baležentis, T. \& Brauers, W., K. (2012). Personnel selection based on computing with words and fuzzy MULTIMOORA.Expert Systems with Applications, 39, 7961-7967.

Bebčáková,I., Holecek, P. \& Talasova, J. (2011). On the application of the fuzzified Choquet integral to multiple criteria evalution.Acta Polytechnica Hungarica, 8(3), 65-78.

Boran, F. E., Genç, S. \& Akay, D. (2011). Personnel selection based on intuitionistic fuzzy sets. Human Factors and Ergonomics in Manufacturing \& Service Industries, 21, 493-503.

Büyüközkan, G., Feyzioğlu, O. \& Göçer, F. (2018). Selection of sustainable urban transportation alternatives using an integrated intuitionistic fuzzy Choquet integral approach. Transportation Research Part D: Transport and Environment, 58, 186-207.

Chen, C.T. (2000). Extensions of the TOPSIS for group decision-making under fuzzy environment. Fuzzy Sets and Systems, 114, 1-9.

Chen, Y. W. \& Tzeng, G. H. (2001). Using fuzzy integral for evaluating subjectively perceived travel costs in a traffic assignment model. European Journal of Operational Research, 130, 653-664.

Chiou, H. K., Tzeng, G. H. \& Cheng, D. C. (2005). Evaluating sustainable fishing development stragtegies using fuzzy MCDM approach. Omega, 33, 223-234.

Delgado, M., Herrera, F., Herrera-Viedma, E. \& Martnez, L. (1998). Combining numerical and linguistic information in group decision making. Information Sciences, 107, 177-194.

Demirel, T., Demirel, N. C. \& Kahraman, C. (2010). Multi criteria warehouse location selection using Choquet integral. Expert Systems with Applications, 37(5), 3943-3952.

Demirel, N. Ç., Demirel, T., Deveci, M. \& Vardar, G. (2017). Location selection for underground natural gasstorage using Choquet integral. Journal of Natural Gas Science and Engineering, 45, 368-379.

Gomesa, L. A., Machado, M. A. S., Rangel, L. A. \& Araujo, R. (2015). Using the Choquet Integral to Improve Systems Usability: A multicriteria analysis. Procedia Computer Science, 31, 606-614.

Grabisch, M.\& Roubens, M. (2000). Application of the Choquet Integral in Multicriteria Decision Making. Fuzzy Measures Integrals, 40, 348-375.

Kabak, M., Burmaoğlu, S. \& Kazançoğlu, Y. (2012). A fuzzy hybrid MCDM approach for professional selection. Expert Systems with Applications, 39, 3516-3525.

Karabasevic, D., Stanujkic, D., Urosevic, S. \& Maksimovic, M. (2015). Selection of candidates in the mining industry based on the application of the SWARA and the MULTIMOORA methods. Acta Montanistica Slovaca, 20, 116-124.

Karsak, E. E. (2005). Choquet integral-based decision making operator for robot selection. Knowledge-based Intelligent Information and Engineering Systems, 3682, 635-641.

Kelemenis, A. \& Askounis, D. (2010). A new TOPSIS based multi-criteria approach to personnel selection. Expert Systems with Applications, 37, 4999-5008.

Li, G., Law, R., Vu, H. Q. \& Rong, J. (2013). Discovering the Hotel Selection Preferences of Hong Kong in Bound Travelers Using the Choquet Integral. Tourism Management, 36, 321-330.

Mazaud, C., Rendek, J., Bombardier, V. \& Wendling, L. A. (2007). A feature selection method based on Choquet integral and typicality analysis. Proc. 16th Int. Conf. FUZZ-IEEE, 1073-1708.

Meyer, P. \& Roubens, M. (2006). On the use of the Choquet integral with fuzzy numbers in multiple criteria decision aiding. Fuzzy Sets and Systems, 157, 927-938.

Nalbant, K. G. (2017). Weighting personnel selection criteria and personnel selection for promotion by using Multi Criteria Decision Making methods (MSc. thesis). Retrieved from https://tez.yok.gov.tr/UlusalTezMerkezi. 
Nia, A. S., Olfat, L., Esmaeili, A., Rostamzadeh, R. \& Antuchevičene, J. (2016). Using fuzzy Choquet integral operator for supplier selection with environmental considerations. Journal of Business Economics and Management, 17, 503-526.

Ozdemir, Y. \& Basligil, H. (2016). Aircraft selection using Fuzzy ANP and the generalized Choquet Integral method: The Turkish Airlines case. Journal of Intelligent and Fuzzy Systems, 31, 589-600.

Ozdemir, Y., Nalbant, K. G. \& Basligil, H. (2017). Evaluation of personnel selection criteria using Consistent Fuzzy Preference Relations. Operations Research and Information Engineering, 2, 1-6.

Rashidi, A., Jazebi, F. \& Brilakis, I. (2011). Neurofuzzy genetic system for selection of construction project managers. Journal of Construction Engineering and Management, 137, 17-29.

Roy, B. \& Misra, S. K. (2012). An integrated DEMATEL and AHP approach for personnel estimation. International Journal of Computer Science and Information Technology \& Security, 2, 1206-1212.

Tan, C. Q. \& Chen, X. H. (2010). Intuitionistic fuzzy Choquet integral operator for multi-criteria decision making. Expert Systems with Applications, 37, 149-157.

Tsai, H. H. \& Lu, I. Y. (2006). The evaluation of service quality using generalized Choquet integral. Inform Sciences, 176, 640-663.

Tseng, M. L., Chiang, J. H. \& Lan, L. W. (2009). Selection of optimal supplier in supply chain management strategy with analytic network process and Choquet Integral. Comput. Ind. Eng., 57, 330-340.

Violeta, K. \& Turskis, Z. (2014). A hybrid linguistic fuzzy multiple criteria group selection of a chief accounting officer. Journal of Business Economics and Management, 15, 232-252.

Wu, J., Chen, F., Cuiping, N. \& Zhang, Q (2013). Intuitionistic fuzzy-valued Choquet integral and its application in multicriteria decision making. Information Sciences, 222, 509-527.

Yu, D., Zhang, W. \& Xu, Y. (2013). Group decision making under hesitant fuzzy environment with application to personnel evaluation. Knowledge Based Systems, 52, 1-10. 